\title{
A novel multi-objective quantum particle swarm algorithm for suspension optimization
}

\author{
Ewerton Grotti ${ }^{1}$ - Douglas Makoto Mizushima ${ }^{1}$. Artur Dieguez Backes ${ }^{1}$. \\ Marcos Daniel de Freitas Awruch ${ }^{1}$. Herbert Martins Gomes ${ }^{1}$ (D
}

Received: 23 April 2019 / Revised: 18 February 2020 / Accepted: 4 March 2020

c) SBMAC - Sociedade Brasileira de Matemática Aplicada e Computacional 2020

\begin{abstract}
In this paper, a novel multi-objective archive-based Quantum Particle Optimizer (MOQPSO) is proposed for solving suspension optimization problems. The algorithm has been adapted from the well-known single objective QPSO by substantial modifications in the core equations and implementation of new multi-objective mechanisms. The novel algorithm MOQPSO and the long-established NSGA-II and COGA-II (Compressed-Objective Genetic Algorithm with Convergence Detection) are compared. Two situations are considered in this paper: a simple half-car suspension model and a bus suspension model. The numerical model of the bus allows complex dynamic interactions not considered in previous studies. The suitability of the solution is evaluated based on vibration-related ISO Standards, and the efficiency of the proposed algorithm is tested by dominance comparison. For a specifically chosen Pareto front solution found by MOQPSO in the second case, the passengers and driver accelerations attenuated about $50 \%$ and $33 \%$, respectively, regarding non-optimal suspension parameters. All solutions found by NSGA-II are dominated by those found by MOQPSO, which presented a Pareto front noticeably wider for the same number of objective function calls.
\end{abstract}

Keywords Dynamics of multibody systems · Computational method stochastic programming $\cdot$ Multi-objective and goal programming

Mathematics Subject Classification 70E55 · 93B40 · 90C15 · 90C29

\section{Introduction}

Vehicle's vibration depends mainly on the vehicle speed, road surface roughness, and suspension system, and is one of the main health issues for bus drivers. Spinal problems are often spotted in long-dated workers, and minor symptoms such as discomfort within passengers, lack of attention, drowsiness, motion sickness, and mental load are also reported as

Communicated by Hector Cancela.

Herbert Martins Gomes

herbert@mecanica.ufrgs.br

1 Federal University of Rio Grande do Sul, Porto Alegre, Rio Grande do Sul, Brazil 
vibration-related problems (Zamanian et al. 2014). The vibration that happens in vehicles can either damage vehicle's components as well as affect road durability or harm the human body. The suspension of the vehicle is mainly designed to mitigate those problems and to promote better driving stability, leading to a safer and more comfortable ride. Although the use of active suspension has grown, nowadays, the passive suspension is still the most costeffective used approach. That being said, to optimize the stiffness and damping parameters that compose the suspension is an important task to be performed and the motivation of this research. This optimization can take into account several objectives that, most of the time, may be conflicting (ride comfort, road-holding, stability, handling, etc.). Hence, a unique solution frequently cannot be attained and Pareto fronts should be used to support an expert in the decision-making process, thus justifying the use of a multi-objective tool.

The main contribution of this paper relies on the proposed new multi-objective algorithm, which shows to be very efficient in solving suspension optimization problems. Objectively, the new algorithm obtains better Pareto Fronts (in terms of range, spread, and dominance) than that found by the traditional NSGA-II in the investigated examples. The novel algorithm shows to be superior when comparing to other advanced algorithms from the literature of vehicle suspension optimization, such as COGA-II.

A secondary innovation concerns the proposal of a complex numerical bus suspension model that allows nonlinear dynamic interactions between degrees of freedom not present in studies with the traditional vertical dynamics only models. The model proposed by Sekulic et al. (2013) is further improved, allowing interactions between the new degrees of freedom: lateral, longitudinal, and yaw. These complex dynamic interactions generate complex behaviors in RMS acceleration of passengers, which opens space for further discussion.

The paper is divided into seven sections: Sect. 1 is the current introduction. Section 2 brings a brief bibliographical review. Section 3 defines the suspension models used in the multi-objective optimization problems and presents all the tools needed to run the numerical simulations. Section 4 explains the new algorithm mechanisms and all proposed enhancements. Section 5 brings a comparison between MOQPSO and COGA-II using the half-car model. Section 6 presents the bus suspension problem, explains the strategy used, and holds a brief discussion on the interpretation of the presented Pareto front graphs. Finally, Sect. 7 summarizes the main conclusions.

\section{Brief bibliographical review}

The multi-objective optimization of vehicle's suspension has attracted the attention of many researchers along this decade (Gomes 2016; Koulocheris et al. 2017; Chen et al. 2015; Florea et al. 2017; Gadhvia et al. 2016; Havelka and Musil 2014). A methodology for designing a hybrid active suspension system was developed by Spentzas and Kanarachos (2002), in which a cost function (passenger comfort) was used to control the passenger's vertical acceleration. Spentzas and Kanarachos (2002) use a computational intelligence approach based on Artificial Neural Network to learn the unknown control function. The vehicle is modeled as 2 degrees of freedom (DOF) model, and the numerical comparisons are performed in the time domain.

Work by Ahmadian et al. (2005) developed an active suspension system to attenuate the acceleration magnitude for the passenger within the threshold limits for human health. Their research considers a quarter-car model with three vibration control approaches: the optimal control, Fuzzy Control, and Fuzzy Optimum Adaptive Control. Large vibration attenuations are obtained with all methods. The topic is still under consideration by several authors like in 
Sert and Boyraz (2017), which used a numeric model to optimize the drive handling in heavy vehicles, increasing the overall roll stiffness of the suspension. Also, Mitra et al. (2016) used a simple numeric model (1/4 car) and a multi-objective Genetic Algorithm (GA) to optimize the suspension to absorb vibrations to comply with ISO 2631-1 (1997) standard limits.

The metaheuristic Quantum Particle Swarm Optimization (QPSO) algorithm was first proposed by Sun et al. (2004). Other authors used the model to solve multi-objective problems like in Omkar et al. (2009) that applied the "vector evaluated" technique to create a simple multi-objective algorithm used in a composite structural problem. That work consists of creating two different populations, which are assigned single objective functions. The population exchange information between themselves was to guide the swarms towards the Pareto front.

QPSO is used in Hassani and Lee (2015) to design state feedback controllers. The proposed algorithm uses an improved initialization method based on the Gaussian neighborhood selection and simulated annealing mechanism, giving a better start point to the individuals of the swarm. An aggregated dynamic weighting criterion is used to solve the multi-objective dilemma which dynamically combines the soft and hard constraints with the control-objectives. The above authors found satisfactory results despite the simplicity of the algorithm.

New approaches to the multi-objective QPSO have been suggested by other authors such as Goh et al. (2012), Zhan and Qing-Wei (2011), and Yang et al. (2013). A novel positionupdate strategy based on a ring model that increases the diversity of the swarm was proposed by Zhou et al. (2016). Changes in the core equation of quantum particle behaved algorithm for the double potential well and sharing learning model affecting directly the used probability density function were proposed by Xu et al. (2016). They claim that this overcomes the deficiency of particles readily gathering in identical solutions, improving the convergence accuracy and diversity.

The nearest application of the QPSO to passive vehicle suspension optimization is presented by Lee and Cheng (2014) which solves the problem of sensitivity assessment in a rail vehicle system with 14 degrees of freedom with nonlinear coupled differential equations of motion.

The use of QPSO as a multi-objective tool to evaluate improvements in general multiobjective optimization problems is reported by Al-Baity (2015) and this approach resembles the method proposed in this paper. The results from Al-Baity (2015) show that the QPSO is a valid tool to multi-objective problems, and can lead to better results in some benchmark functions than other famous algorithms such as NSGA-II (Deb et al. 2002), PAEAS, and SPEA2. Recently, Fan et al. (2017) present a paper where a new MOPSO is proposed based on the minimum distance of point-to-line called MDPL-MOPSO. The algorithm is tested with the well-known ZDT multi-objective functions and with a half-car suspension model with multi-objective functions such as acceleration, suspension stroke, and velocity.

The five degrees of freedom half-car suspension model is used in the first case of the present work and was previously analyzed by Fan et al. (2017), Nariman-Zadeh et al. (2010), Mahmoodabadi et al. (2013), and Boonlong (2013) using different multi-objective optimization algorithms. The use of the Multi-objective Uniform-diversity Genetic Algorithm (MUGA) with a diversity mechanism called the $\varepsilon$-elimination algorithm is used by Nariman-Zadeh et al. (2010) to optimize four different pairs of objective functions and a set of five conflicting objective functions at the same time in the 5 DOF half-car problem. These results are incorporated by Boonlong (2013) that proposes the Improved Compressed-Objective Genetic Algorithm (COGA-II) to compare the solutions obtained by Nariman-Zadeh et al. (2010) and reports that the results were superior. 
The present article proposes a metaheuristic algorithm of multi-objective optimization based on the behavior of quantum particles (QPSO) applied to the suspension optimization problem of a bus, named Multi-objective Quantum Particle Swarm Optimization (MOQPSO). First, the algorithm is put to test in the aforementioned half-car suspension model for comparison with the COGA-II algorithm. Finally, a bus model that accounts for vertical and lateral dynamic behavior in a standardized double lane change maneuver serves as a test case for the MOQPSO. The inclusion of lateral dynamics coupled with the differential equations of vertical dynamics is new and the set of the equation is solved in time domain by the nonlinear Newmark method. As a secondary goal, the results obtained are comparatively evaluated with those obtained by NSGA-II, a well-known multi-objective algorithm.

The main motivation of the paper relies on the better performance obtained with the new algorithm for multi-objective optimization using MOQPSO and newly proposed metaheuristic that allows obtaining Pareto fronts that are more spread and advanced than that using traditional well-established Multi-objective optimization tools like NSGA-II. The dynamic model is an improved version of the vertical only model presented by Sekulic et al. (2013). There is no previous work in the authors' knowledge that uses such a complex model in parametric multi-objective optimization. That alone leads to the investigation of the consequences in the optimization by introducing such degrees of freedom and complex interactions.

\section{Numerical models}

\subsection{Half-car numeric model}

The numerical model used for the half-car suspension system is depicted in Fig. 1, and Eqs. 1-5. This is the same model used by Boonlong (2013) for the sake of comparison. The dynamic equations of motion for each DOF are defined as:

$$
\begin{gathered}
m_{1} \ddot{z}_{1}=-k_{\mathrm{p} 1}\left(z_{1}-\xi_{1}\right)-c_{\mathrm{p} 1}\left(\dot{z}_{1}-\dot{\xi}_{1}\right)+k_{\mathrm{s} 1}\left(z_{\mathrm{s} 1}-z_{1}\right)+c_{\mathrm{s} 1}\left(\dot{z}_{\mathrm{s} 1}-\dot{z}_{1}\right), \\
m_{2} \ddot{z}_{2}=-k_{\mathrm{p} 2}\left(z_{2}-\xi_{2}\right)-c_{\mathrm{p} 2}\left(\dot{z}_{2}-\dot{\xi}_{2}\right)+k_{\mathrm{s} 2}\left(z_{\mathrm{s} 2}-z_{2}\right)+c_{\mathrm{s} 2}\left(\dot{z}_{\mathrm{s} 2}-\dot{z}_{2}\right), \\
m_{\mathrm{s}} \ddot{z}_{\mathrm{s}}=-k_{\mathrm{s} 1}\left(z_{\mathrm{s} 1}-z_{1}\right)-c_{\mathrm{s} 1}\left(\dot{z}_{\mathrm{s} 1}-\dot{z}_{1}\right)-k_{\mathrm{s} 2}\left(z_{\mathrm{s} 2}-z_{2}\right)-c_{\mathrm{s} 2}\left(\dot{z}_{\mathrm{s} 2}-\dot{z}_{2}\right) \\
+k_{\mathrm{ss}}\left(z_{\mathrm{c}}-z_{\mathrm{sp}}\right)+c_{\mathrm{s} 2}\left(\dot{z}_{\mathrm{c}}-\dot{z}_{\mathrm{sp}}\right), \\
m_{\mathrm{c}} \ddot{z}_{\mathrm{c}}=-k_{\mathrm{ss}}\left(z_{\mathrm{c}}-z_{\mathrm{sp}}\right)-c_{\mathrm{ss}}\left(\dot{z}_{\mathrm{c}}-\dot{z}_{\mathrm{sp}}\right), \\
J_{\mathrm{s}} \ddot{\theta}_{\mathrm{s}}=-k_{\mathrm{ss}}\left(z_{\mathrm{c}}-z_{\mathrm{sp}}\right) r-c_{\mathrm{ss}}\left(\dot{z}_{\mathrm{c}}-\dot{z}_{\mathrm{sp}}\right) r+k_{\mathrm{s} 1}\left(z_{\mathrm{s} 1}-z_{1}\right) L_{1}+c_{\mathrm{s} 1}\left(\dot{z}_{\mathrm{s} 1}-\dot{z}_{1}\right) L_{1} \\
-k_{\mathrm{s} 2}\left(z_{\mathrm{s} 2}-z_{2}\right) L_{2}-c_{\mathrm{s} 2}\left(\dot{z}_{\mathrm{s} 2}-\dot{z}_{2}\right) L_{2} .
\end{gathered}
$$

Newmark's linear method is used to solve the set of coupled equations in the time domain. The vehicle moves forward through a constant speed of $20 \mathrm{~m} / \mathrm{s}$. The model input is the road profile at each tire, simulated as a double bump in a simple sinusoidal format with an amplitude of $0.05 \mathrm{~m}$ and a period of $1 \mathrm{~s}$.

In Fig. $1, z_{\mathrm{s} 1}=z_{\mathrm{s}}-\sin \left(\theta_{\mathrm{s}}\right) L_{1}, z_{\mathrm{s} 2}=z_{\mathrm{s}}+\sin \left(\theta_{\mathrm{s}}\right) L_{2}$, and $z_{\mathrm{sp}}=z_{\mathrm{s}}-\sin \left(\theta_{\mathrm{s}}\right) r$.

\subsection{Bus numeric model}

The bus model is an improvement of the IK-301 bus model described in Sekulic et al. (2013). As described in the paper, two airbags and four telescopic shock absorbers attach the front 


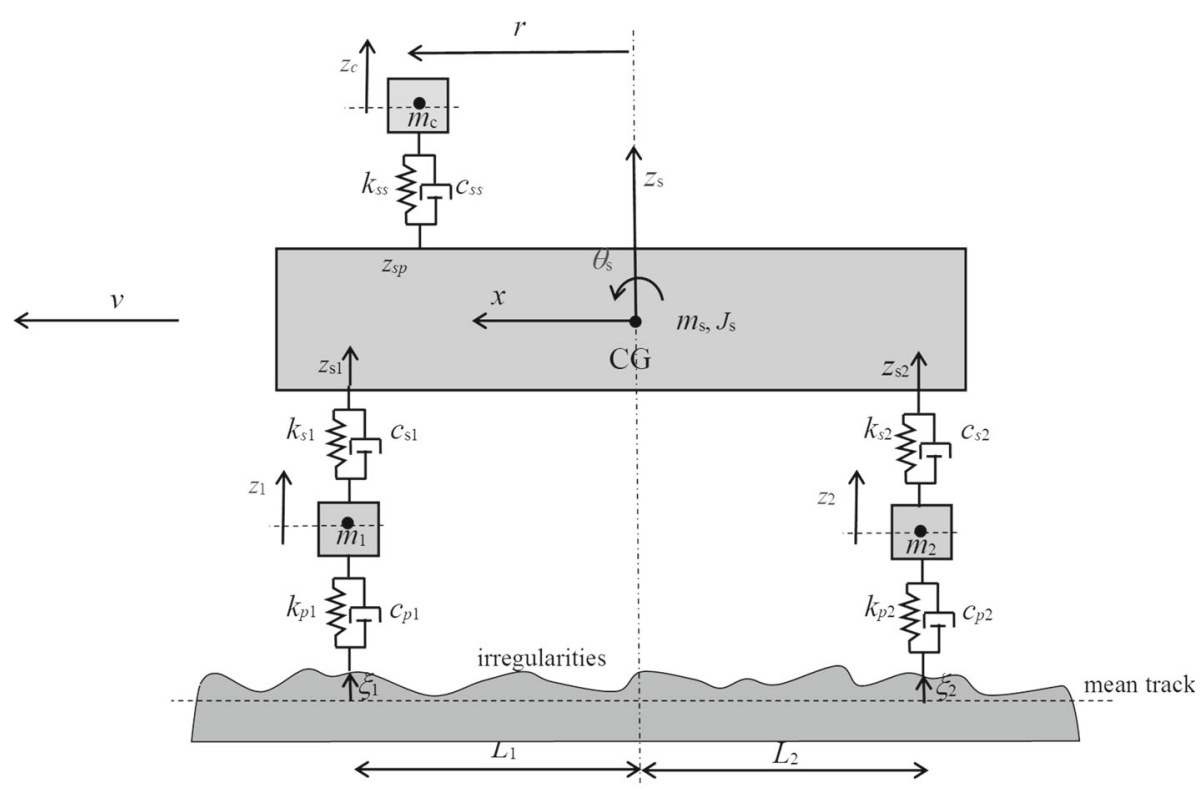

Fig. 1 Sketch of the half-car suspension model

stiff axle to the bus bodywork, while the rear axle connects to the bus bodywork by four airbags and four telescopic shock absorbers. The bus has two wheels mounted on the front axle and four wheels on the rear one. The proposed model extends this model by adding 3 new degrees of freedom for lateral, yaw, and longitudinal dynamics. The model was validated by Sekulic et al. (2013) against experimental values (with the actual bus at a constant speed and driving straight) for vertical acceleration and presented acceptable concordance with the measured data. Therefore, the proposed extended model in this paper was validated at a constant speed and driving straight, where experimental data are available and presented acceptable concordance as in Sekulic et al. (2013). The coupled equations for the 13 DOF suspension bus model are described as follows:

Driver's vertical DOF:

$$
m_{\mathrm{v}} \ddot{z}_{\mathrm{v}}+c_{\mathrm{sv}} \dot{z}_{\mathrm{v}}+k_{\mathrm{sv}} z_{\mathrm{v}}-c_{\mathrm{sv}} \dot{z}-k_{\mathrm{sv}} z-s_{1} c_{\mathrm{sv}} \dot{\varphi}-s_{1} k_{\mathrm{sv}} \varphi+s_{2} c_{\mathrm{sv}} \dot{\theta}+s_{2} k_{\mathrm{sv}} \theta=0 .
$$

Passenger 1 and Passenger 2 vertical DOF:

$$
\begin{gathered}
m_{\mathrm{p} 1} \ddot{z}_{\mathrm{p} 1}+c_{\mathrm{sp} 1} \dot{z}_{\mathrm{p} 1}+k_{\mathrm{sp} 1} z_{\mathrm{p} 1}-c_{\mathrm{sp} 1} \dot{z}-k_{\mathrm{sp} 1} z+s_{3} c_{\mathrm{sp} 1} \dot{\varphi}+s_{3} k_{\mathrm{sp} 1} \varphi+s_{4} c_{\mathrm{sp} 1} \dot{\theta}+s_{4} k_{\mathrm{sp} 1} \theta=0 . \\
m_{\mathrm{p} 2} \ddot{z}_{\mathrm{p} 2}+c_{\mathrm{sp} 2} \dot{z}_{\mathrm{p} 2}+k_{\mathrm{sp} 2} z_{\mathrm{p} 2}-c_{\mathrm{sp} 2} \dot{z}-k_{\mathrm{sp} 2} z-s_{5} c_{\mathrm{sp} 2} \dot{\varphi}-s_{5} k_{\mathrm{sp} 2} \varphi-s_{6} c_{\mathrm{sp} 2} \dot{\theta}-s_{6} k_{\mathrm{sp} 2} \theta=0 .
\end{gathered}
$$

Bus body vertical DOF:

$$
\begin{aligned}
m \ddot{z} & +\left(c_{\mathrm{sv}}+c_{\mathrm{sp} 1}+c_{\mathrm{sp} 2}+2 c_{\mathrm{p}}+2 c_{\mathrm{z}}\right) \dot{z}+\left(k_{\mathrm{sv}}+k_{\mathrm{sp} 1}+k_{\mathrm{sp} 2}+2 k_{\mathrm{p}}+2 k_{\mathrm{z}}\right) z \\
& +\left(s_{1} c_{\mathrm{sv}}-s_{3} c_{\mathrm{sp} 1}+s_{5} c_{\mathrm{sp} 2}\right) \dot{\varphi}+\left(s_{1} k_{\mathrm{sv}}-s_{3} k_{\mathrm{sp} 1}+s_{5} k_{\mathrm{sp} 2}\right) \varphi \\
& -\left(s_{2} c_{\mathrm{sv}}+s_{4} c_{\mathrm{sp} 1}-s_{6} c_{\mathrm{sp} 2}+2 a c_{\mathrm{p}}-2 b c_{\mathrm{z}}\right) \dot{\theta}-\left(s_{2} k_{\mathrm{sv}}+s_{4} k_{\mathrm{sp} 1}-s_{6} k_{\mathrm{sp} 2}+2 a k_{\mathrm{p}}-2 b k_{\mathrm{z}}\right) \theta-c_{\mathrm{sv}} \dot{z}_{\mathrm{v}} \\
& -k_{\mathrm{sv}} z_{\mathrm{v}}-c_{\mathrm{sp} 1} \dot{z}_{\mathrm{p} 1}-k_{\mathrm{sp} 1} z_{\mathrm{p} 1}-c_{\mathrm{sp} 2} \dot{z}_{\mathrm{p} 2}-k_{\mathrm{sp} 2} z_{\mathrm{p} 2}-2 c_{\mathrm{p}} \dot{z}_{1}-2 k_{\mathrm{p}} z_{1}-2 c_{\mathrm{z}} \dot{z}_{2}-2 k_{\mathrm{z}} z_{2}=0
\end{aligned}
$$




\section{Roll DOF:}

$$
\begin{aligned}
J_{x} \ddot{\varphi}+ & \left(s_{1}^{2} c_{\mathrm{sv}}+s_{3}^{2} c_{\mathrm{sp} 1}+s_{5}^{2} c_{\mathrm{sp} 2}+2 e_{1}^{2} c_{\mathrm{p}}+2 e_{2}^{2} c_{\mathrm{z}}\right) \dot{\varphi}+\left(s_{1}^{2} k_{\mathrm{sv}}+s_{3}^{2} k_{\mathrm{sp} 1}+s_{5}^{2} k_{\mathrm{sp} 2}+2 e_{1}^{2} k_{\mathrm{p}}+2 e_{2}^{2} k_{\mathrm{z}}\right) \varphi \\
& -s_{1} c_{\mathrm{sv}} \dot{z}_{\mathrm{v}}-s_{1} k_{\mathrm{sv}} z_{\mathrm{v}}+s_{3} c_{\mathrm{sp} 1} \dot{z}_{\mathrm{p} 1}+s_{3} k_{\mathrm{sp} 1} z_{\mathrm{p} 1}-s_{5} k_{\mathrm{sp} 2} z_{\mathrm{p} 2}-s_{5} c_{\mathrm{sp} 2} \dot{z}_{\mathrm{p} 2} \\
& +\left(s_{1} c_{\mathrm{sv}}-s_{3} c_{\mathrm{sp} 1}+s_{5} c_{\mathrm{sp} 2}\right) \dot{z}+\left(s_{1} k_{\mathrm{sv}}-s_{3} k_{\mathrm{sp} 1}+s_{5} k_{\mathrm{sp} 2}\right) z \\
& -\left(s_{1} s_{2} c_{\mathrm{sv}}-s_{3} s_{4} c_{\mathrm{sp} 1}-s_{5} s_{6} c_{\mathrm{sp} 2}\right) \dot{\theta}-\left(s_{1} s_{2} k_{\mathrm{sv}}-s_{3} s_{4} k_{\mathrm{sp} 1}-s_{5} s_{6} k_{\mathrm{sp} 2}\right) \theta-2 e_{1}^{2} c_{\mathrm{p}} \dot{\varphi}_{1} \\
& -2 e_{1}^{2} k_{\mathrm{p}} \varphi_{1}-2 e_{2}^{2} c_{z} \dot{\varphi}_{2}-2 e_{2}^{2} k_{z} \varphi_{2}=0 .
\end{aligned}
$$

Pitch DOF:

$$
\begin{aligned}
J_{y} \ddot{\theta} & +\left(s_{2}^{2} c_{\mathrm{sv}}+s_{4}^{2} c_{\mathrm{sp} 1}+s_{6}^{2} c_{\mathrm{sp} 2}+2 a^{2} c_{\mathrm{p}}+2 b^{2} c_{\mathrm{z}}\right) \dot{\theta}+\left(s_{2}^{2} k_{\mathrm{sv}}+s_{4}^{2} k_{\mathrm{sp} 1}+s_{6}^{2} k_{\mathrm{sp} 2}+2 a^{2} k_{\mathrm{p}}+2 b^{2} k_{\mathrm{z}}\right) \theta \\
& +s_{2} c_{\mathrm{sv}} \dot{z}_{\mathrm{v}}+s_{2} k_{\mathrm{sv}} z_{\mathrm{v}}+s_{4} c_{\mathrm{sp} 1} \dot{z}_{\mathrm{p} 1}+s_{4} k_{\mathrm{sp} 1} z_{\mathrm{p} 1}-s_{6} k_{\mathrm{sp} 2} z_{\mathrm{p} 2}-s_{6} c_{\mathrm{sp} 2} \dot{z}_{\mathrm{p} 2} \\
& -\left(s_{2} c_{\mathrm{sv}}+s_{4} c_{\mathrm{sp} 1}-s_{6} c_{\mathrm{sp} 2}+2 a c_{\mathrm{p}}-2 b c_{\mathrm{z}}\right) \dot{z} \\
& -\left(s_{1} s_{2} c_{\mathrm{sv}}-s_{3} s_{4} c_{\mathrm{sp} 1}-s_{5} s_{6} c_{\mathrm{sp} 2}\right) \dot{\varphi}-\left(s_{2} k_{\mathrm{sv}}+s_{4} k_{\mathrm{sp} 1}-s_{6} k_{\mathrm{sp} 2}+2 a k_{\mathrm{p}}-2 b k_{\mathrm{z}}\right) z \\
& -\left(s_{1} s_{2} k_{\mathrm{sv}}-s_{3} s_{4} k_{\mathrm{sp} 1}-s_{5} s_{6} k_{\mathrm{sp} 2}\right) \varphi+2 a c_{\mathrm{p}} \dot{z}_{1}+2 a k_{\mathrm{p}} z_{1}-2 b c_{\mathrm{z}} \dot{z}_{2}-2 b k_{\mathrm{z}} z_{2}=0 .
\end{aligned}
$$

Front axis vertical DOF:

$$
\begin{aligned}
& m_{\mathrm{pm}} \ddot{z}_{1}+2\left(c_{\mathrm{p}}+c_{\mathrm{pp}}\right) \dot{z}_{1}+2\left(k_{\mathrm{p}}+k_{\mathrm{pp}}\right) z_{1}-2 c_{\mathrm{p}} \dot{z}-2 k_{\mathrm{p}} z \\
& \quad+2 a c_{\mathrm{p}} \dot{\theta}+2 a k_{\mathrm{p}} \theta-c_{\mathrm{pp}} \dot{\xi}_{\mathrm{pd}}-k_{\mathrm{pp}} \xi_{\mathrm{pd}}-c_{\mathrm{pp}} \dot{\xi}_{\mathrm{pl}}-k_{\mathrm{pp}} \xi_{\mathrm{pl}}=0 .
\end{aligned}
$$

Front axis roll DOF:

$$
\begin{aligned}
& J_{x 1} \ddot{\varphi}_{1}+2\left(e_{1}^{2} c_{\mathrm{p}}+f_{1}^{2} c_{\mathrm{pp}}\right) \dot{\varphi}_{1}+2\left(e_{1}^{2} k_{\mathrm{p}}+f_{1}^{2} k_{\mathrm{pp}}\right) \varphi_{1}-2 e_{1}^{2} c_{\mathrm{p}} \dot{\varphi}-2 e_{1}^{2} k_{\mathrm{p}} \varphi+f_{1} c_{\mathrm{pp}} \dot{\xi}_{\mathrm{pd}} \\
& \quad+f_{1} k_{\mathrm{pp}} \xi_{\mathrm{pd}}-f_{1} c_{\mathrm{pp}} \dot{\xi}_{\mathrm{pl}}-f_{1} k_{\mathrm{pp}} \xi_{\mathrm{pl}}=0 .
\end{aligned}
$$

Rear axis vertical DOF:

$$
\begin{aligned}
& m_{\mathrm{zm}} \ddot{z}_{2}+2\left(c_{\mathrm{z}}+c_{\mathrm{zp}}\right) \dot{z}_{2}+2\left(k_{\mathrm{z}}+k_{\mathrm{zp}}\right) z_{2}-2 c_{\mathrm{z}} \dot{z}-2 k_{\mathrm{z}} z-2 b c_{\mathrm{z}} \dot{\theta}-2 b k_{\mathrm{z}} \theta-c_{\mathrm{zp}} \dot{\xi}_{\mathrm{zd}} \\
& \quad-k_{\mathrm{zp}} \xi_{\mathrm{zd}}-c_{\mathrm{zp}} \dot{\xi}_{\mathrm{zl}}-k_{\mathrm{zp}} \xi_{\mathrm{zl}}=0 .
\end{aligned}
$$

Rear axis roll DOF:

$$
\begin{aligned}
& J_{x 2} \ddot{\varphi}_{2}+2\left(e_{2}^{2} c_{\mathrm{z}}+f_{2}^{2} c_{\mathrm{zp}}\right) \dot{\varphi}_{2}+2\left(e_{2}^{2} k_{\mathrm{z}}+f_{2}^{2} k_{\mathrm{zp}}\right) \varphi_{2}-2 e_{2}^{2} c_{\mathrm{z}} \dot{\varphi}-2 e_{2}^{2} k_{\mathrm{z}} \varphi+f_{2} c_{\mathrm{zp}} \dot{\xi}_{\mathrm{zd}} \\
& \quad+f_{2} k_{\mathrm{zp}} \xi_{\mathrm{zd}}-f_{2} c_{\mathrm{zp}} \dot{\xi}_{\mathrm{zl}}-f_{2} k_{\mathrm{zp}} \xi_{\mathrm{zl}}=0 .
\end{aligned}
$$

According to Rill (2012), for the lateral, longitudinal, and yaw DOF, the equations of motion that are coupled with the previous ones are described by:

Lateral DOF:

$$
m \ddot{y}=F_{y 1} \cos \delta_{1}+F_{y 2} \cos \delta_{2}+F_{x 1} \sin \delta_{1}+F_{x 2} \sin \delta_{2}+F_{y 3}+F_{y 4}-m_{\mathrm{zm}} \dot{x} \dot{\psi}-m h \ddot{\varphi},
$$

where $\ddot{y}$ is the lateral acceleration, $\dot{x}$ the component of the longitudinal vehicle's speed, and $\dot{\psi}$, the yaw rate. The term $m h \ddot{\varphi}$ accounts for the influence of roll into the lateral DOF.

Yaw DOF:

$$
\begin{aligned}
J_{z} \ddot{\psi} & =f_{2} F_{x 3}-f_{2} F_{x 4}+f_{1} F_{y 1} \sin \delta_{2}-f_{1} F_{y 2} \sin \delta_{2}-b F_{y 4}-b F_{y 3}+f_{1} F_{x 1} \cos \delta_{1} \\
& -f_{1} F_{x 2} \cos \delta_{2}-a F_{x 1} \sin \delta_{1}-a F_{x 2} \sin \delta_{2}+a F_{y 1} \cos \delta_{1}+a F_{y 2} \cos \delta_{2} .
\end{aligned}
$$




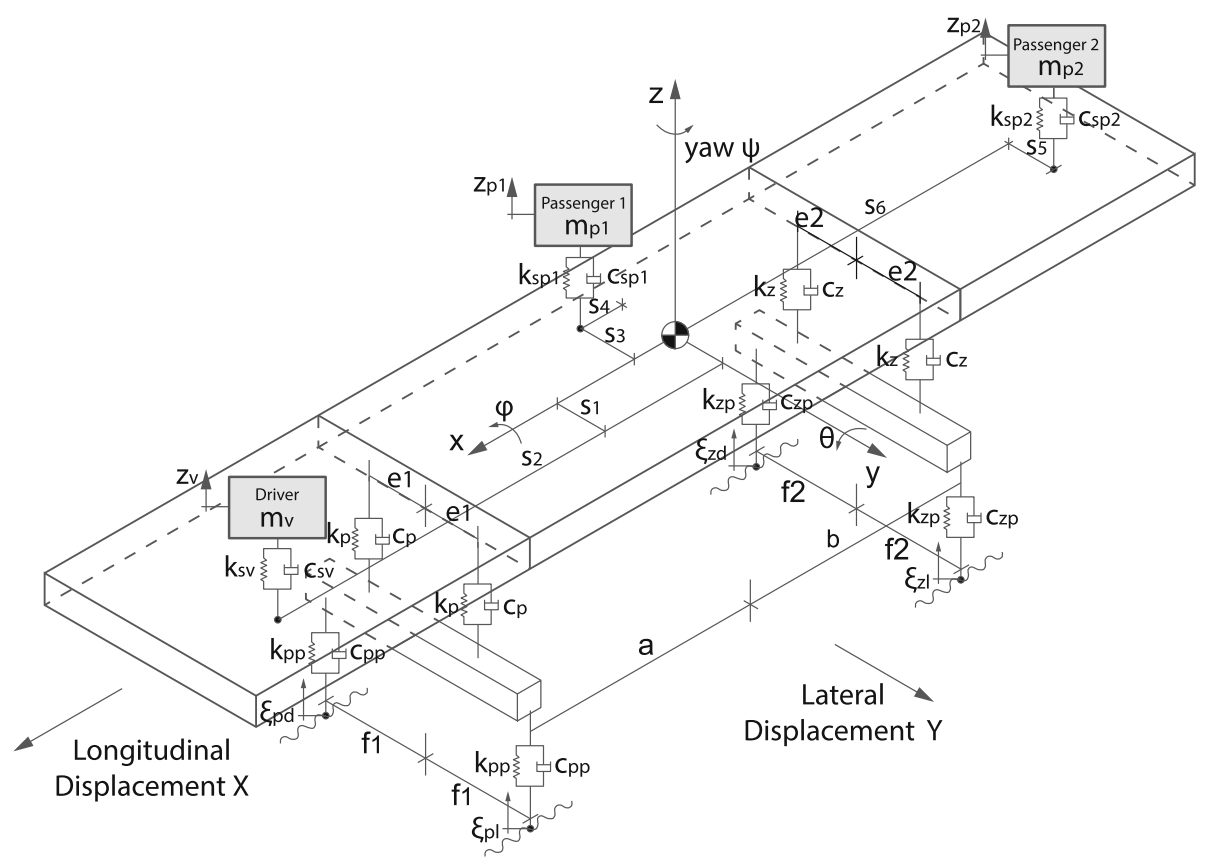

Fig. 2 Bus model with 13 degrees of freedom (adapted from Sekulic et al. (2013))

\section{Longitudinal DOF:}

$m \ddot{x}=F_{x 2} \cos \delta_{2}+F_{x 1} \cos \delta_{1}-F_{y 1} \sin \delta_{1}-F_{y 2} \sin \delta_{2}+F_{x 3}+F_{x 4}+m_{z m} \dot{y} \dot{\psi}+m h \varphi \ddot{\psi}-m h \dot{\varphi} \dot{\psi}$.

In Eqs. (16)-(18), $F_{x i}$ and $F_{y i}$ with $i=1$ to 4 are the $x$ and $y$ local contact tire forces (front left and right, rear left and right), and $\delta_{1}$ and $\delta_{2}$ are the steering angle at each front tire (left and right). The total mass of the vehicle is represented by $m_{z m}$ and $m$ means the sprung mass.

The model is composed of the main chassis with two suspension bars and the seats for a driver and two passengers. The tires are connected by the suspension bars, which, in turn, are connected to the chassis. Figure 2 shows the proposed IK-301bus 3D dynamic model. There are 13 degrees of freedom: rotation of the rear and front axles $\left(\varphi_{1}, \varphi_{2}\right)$, displacements of rear and front axles $\left(z_{1}, z_{2}\right)$, pitch $(\theta)$, roll $(\varphi)$ and vertical displacement of the bus bodywork $(z)$, lateral $(y)$ and longitudinal $(x)$ displacements, yaw $(\psi)$ and the vertical displacements of a seated driver $\left(z_{\mathrm{v}}\right)$, and two seated passengers $\left(z_{\mathrm{p} 1}, z_{\mathrm{p} 2}\right)$. The vertical displacements of the four wheels $\left(z_{\mathrm{w} 1}, z_{\mathrm{w} 2}, z_{\mathrm{w} 3}, z_{\mathrm{w} 4}\right)$ are linked to the rotations and translational DOF of the rear and front axle, similar to those presented in the half-car model $\left(z_{\mathrm{s} 1}, z_{\mathrm{s} 2}, z_{\mathrm{sp}}\right)$. Tables 3 , 4 show the meaning of each variable used.

As mentioned, for the complete bus suspension model, the input is the steering angles $\delta_{i}$ at two front wheels and a simple tire model based on the magic formula is used to account for contact forces during maneuvering. Based on vertical forces at each tire $\left(F_{z j}\right)$, the magic formula evaluates each local lateral and longitudinal tire forces that are used in the lateral dynamics, and this adds another level of coupling with the vertical dynamics, besides the previously mentioned common degrees of freedom in the equations. A nonlinear Newmark algorithm is used to solve the set of nonlinear coupled differential equations. The parameter 


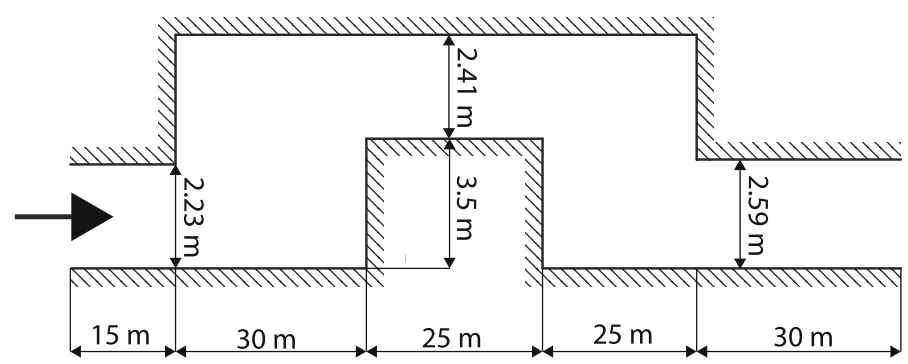

Fig. 3 Typical double lane change geometric parameters scheme for a ride vehicle Adapted from ISO (1999)

values are presented in the numerical results section. Depending on the scale level of analysis, not only the nonlinear analysis for the coupled bus suspension model is advisable, but also the inclusion of a nonlinear tire model. For optimization purposes, the coupled linear system for vertical and lateral dynamics, excited by the DLC maneuver, will activate these nonlinear effects.

Although ISO 5982 (2001) suggests human body models to accommodate their dynamic behavior, using ISO 2631-1 (1997) to evaluate comfort and health risk to vibration does not require the use of such body models. The measurement of vibration at the surface between the vehicle and the human body is enough to comply with ISO 2631-1 (1997). In the case of a heavy vehicle, the interaction between seated human body dynamics (small mass) with the vehicle (large mass) is negligible, and so, modeling the vertical seat dynamics with only a human body mass and evaluating the seat surface acceleration are enough for comfort and health risk estimates.

\subsection{Double lane change maneuver (DLC)}

The double lane change maneuver is a subjective test originally created to simulate a real-life traffic maneuver. It consists of quickly driving from the driver's initial lane to a parallel lane and then returning to the driver's initial lane without exceeding the boundaries at a constant speed of $80 \pm 3 \mathrm{~km} / \mathrm{h}$. The specifications for the maneuver are presented in ISO 3888-1 (1999) and depend on the dimension of the vehicle. The test can be modified to accomplish particular needs. Figure 3 shows a schematic of a typical DLC track for a ride vehicle. For a DLC maneuver and for health risk and comfort analysis, a time-history solution is necessary.

The three degrees of freedom added to the model allow the numerical model to perform lateral movements and simulate the DLC maneuver that will be the focus for the optimization.

\subsection{Road profile}

The road surface is modeled as a single stochastic process with a Power Spectral Density function given by ISO 8608 Standard (1995). It can be stated by Eq. (19):

$$
G_{\xi}(\Omega)= \begin{cases}C\left(\frac{n}{n_{0}}\right)^{-w_{1}} & \text { for } n \leq n_{0} \\ C\left(\frac{n}{n_{0}}\right)^{-w_{2}} & \text { for } n \geq n_{0},\end{cases}
$$


where $C$ is the general road roughness coefficient ( $\mathrm{m}^{3} /$ cycle), which is related to the road surface condition, and $\mathrm{w}$ is the wavelength distribution. $n$ means the wavenumber $(\mathrm{cycle} / \mathrm{m})$; $G_{\xi}$ is the single-sided power spectral density for road roughness $\left(\mathrm{m}^{2} /\right.$ cycle $\left./ \mathrm{m}\right)$. The singlesided PSD is split into two parts at the discontinuity frequency $n_{0}$ (cycle $\left./ \mathrm{m}\right)$. The discontinuity frequency is typically set as $n_{0}=1 / 2 \pi=0.16 \mathrm{cycle} / \mathrm{m} . w_{1}$ and $w_{2}$ are wavelength distribution parameters. The simple model is used in this paper with $n_{0}=0.1 \mathrm{cycle} / \mathrm{m}$, distribution parameter $w_{1}=w_{2}=2.0$, and the general road roughness coefficient $C=0.01 \mathrm{~m}^{3} /$ cycle.

The time signal generation is performed according to a given single-sided PSD by the equation:

$$
x_{a}\left(t_{i}\right)=\sum_{i=1}^{\mathrm{ne}} \sqrt{G_{a}\left(f_{i}\right) \Delta f} \sin \left(2 \pi f_{i} t_{i}+\varphi_{i}\right),
$$

where $\varphi_{i}$ are uncorrelated phase angles uniformly distributed in the range 0 to $2 \pi ; f_{i}$ are the frequencies from the spectral density (discretized by ne spectral lines). A simple phase shift is used to correlate front and rear tracks as usual $G_{a \text {, shift }}(f)=G_{a}(f) e^{i \omega \Delta t}$, where $\Delta t=$ $(a+b) / v$ takes into account the distance between the front and rear axles $(a+b)$ and vehicle speed $(v)$.

To correlate left and right tracks, a widely used cross-correlation model is applied to the spectral densities to be correlated. It is defined for a given cut-off frequency $f_{\mathrm{c}}=0.2 \mathrm{~m}^{-1}$. Therefore, for two parallel tracks, $a$ and $b$ hold:

$$
G_{b}(f)=G_{a}^{*}(f)-\gamma(f) G_{a}^{*}(f)+\gamma(f) G_{a}(f),
$$

where $\gamma(f)=f_{\mathrm{c}}^{2} /\left[f_{\mathrm{c}}^{2}+\left(\frac{f}{v}\right)^{2}\right]$ is the low-pass filter, and $f_{\mathrm{c}}$ is the cut-off frequency which is applied to the PSD that is to be correlated. $G_{a}^{*}(f)$ is the PSD. The time signal will be generated based on this PSD with a different phase angle $\psi_{i}$ uncorrelated from the phase angles $\varphi_{i}$ used to define $G_{a}$. The same Eq. (20) is applied to obtain the opposite correlated track $x_{b}\left(t_{i}\right)$ using $G_{b}$ instead of $G_{a}$. At this point, it should be emphasized the need for assuring the same road irregularities, despite being random, during all the optimization simulations, to obtain fair comparisons between optimized candidate solutions.

\subsection{Whole-body vibration according to ISO 2631-1 (1997)}

The vibration received by the body can be classified, according to ISO 2631-1 (1997), into two main groups: whole-body vibration (WBV) and hand-arm vibration (HAV). The exposure to vibration can generate unnecessary risks (such as headache in reading, dizziness, lack of attention in dangerous or highly responsible activities, etc.) and even harm depending on the frequency range, intensity, and the exposure time. In some moderate situations, this can be a source of discomfort.

Some concern on the effects of WBV (acceleration frequencies ranging from 0.1 to $80 \mathrm{~Hz}$ ) is reported in recent literature surveys, mainly on vehicle seats regarding passenger comfort. Works of Zhang et al. (2015) and Ji et al. (2017) and particularly (Nawayseh 2015) investigate the effect in transmissibility of vibration in heavy vehicles and cars by the foam and air in seat cushions. Sammonds et al. (2017) indicates that there is a current concern in the study of materials and solutions to mitigate passenger vibrations (referring to the effect of discomfort on drivers), especially when driving and exposed to long periods.

The vibration amplitude may be evaluated by the root-mean-square (RMS) acceleration, which gives the harmful potential of the vibration by the average energy contained in the

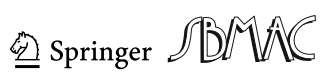


Table 1 Vibration value limits for health and comfort, (ISO 2631 1997)

\begin{tabular}{lll}
\hline & $a_{\text {rms }}$ & VDV \\
\hline Health (ISO 2631-1 1997) & & \\
Exposure action value (8 h): EAV & $0.5 \mathrm{~m} / \mathrm{s}^{2}$ & $8.5 \mathrm{~m} / \mathrm{s}^{1.75}$ \\
Exposure limit value (8 h): ELV & $1.0 \mathrm{~m} / \mathrm{s}^{2}$ & $17.0 \mathrm{~m} / \mathrm{s}^{1.75}$ \\
Comfort (ISO 2361-1 1997) & & \\
Not uncomfortable & $<0.315 \mathrm{~m} / \mathrm{s}^{2}$ \\
A little uncomfortable & $0.315-0.63 \mathrm{~m} / \mathrm{s}^{2}$ \\
Fairly uncomfortable & $0.5-1.0 \mathrm{~m} / \mathrm{s}^{2}$ \\
Uncomfortable & $0.8-1.6 \mathrm{~m} / \mathrm{s}^{2}$ \\
Very uncomfortable & $1.25-2.5 \mathrm{~m} / \mathrm{s}^{2}$ \\
Extremely uncomfortable & $>2.0 \mathrm{~m} / \mathrm{s}^{2}$
\end{tabular}

${ }^{\mathrm{a}}$ Implicit value from figures for a daily exposure time of $8 \mathrm{~h}$ using Eq. 1 from ISO standard

oscillatory movement. The RMS value can be obtained for an acceleration signal $a(t)$, defined in the time interval $t_{1} \leq t \leq t_{2}$, by:

$$
a_{\mathrm{rms}}=\sqrt{\frac{1}{t_{2}-t_{1}} \int_{t_{1}}^{t_{2}}[a(t)]^{2} \mathrm{~d} t .}
$$

ISO 2631-1 (1997) classifies the consequence of vibration in the human body, according to the exposure time and magnitude. For small magnitudes, the main concern is related to comfort. Not only the magnitude of oscillatory vibration may cause damage but also their frequency content (Griffin 1990). For both health and comfort studies, the standard ISO 2631-1 (1997) recommends using the frequency-weighted RMS acceleration, described in Eq. (23):

$$
a_{w}=\left[\sum_{i}\left(w_{i} a_{i}\right)^{2}\right]^{1 / 2},
$$

where $a_{w}$ is the frequency-weighted acceleration, $w_{i}$ is the weighting factor for the $i$ th onethird octave band, and $a_{i}$ is the RMS acceleration for the $i$ th one-third octave band. Table 1 shows the main vibration values limits for health and comfort defined by ISO 2631-1 (1997). In case the crest factor (instantaneous acceleration peak value divided by the RMS value) is above 9 , it is recommended to evaluate the harmful effect of impact vibration.

\section{Optimization algorithm}

\subsection{Quantum particle swarm optimization (QPSO)}

According to Sun et al. (2004a, b, 2011), the QPSO has shown interesting characteristics when compared to other heuristics:

- it has great exploratory tendencies, which allows for the algorithm to search through the design space without getting stuck in local minima. This trait, in particular, is very useful for solving chaotic behaved problems; 
- it has a simpler concept and equations, being easier to implement and adjust to each problem;

- in the single objective domain, it has promising results, but in the multi-objective domain, the QPSO is not yet well investigated in the literature;

- According to Yang et al. (2013), QPSO has a better convergence behavior than PSO.

The investigation of the QPSO as a multi-objective tool is attractive, and the pointed above features make it well fit for solving the optimization problem of complex suspension models, justifying the choice for the algorithm. The QPSO was developed by Sun et al. (2004) using the traditional Particle Swarm Optimization method (PSO) as the starting point for the QPSO. In the PSO, the particles are updated using position and velocity. The QPSO, however, update the particles using the square of the module of a wave function $|\psi(x, t)|^{2}$, that being the probability density function of particle's positions. Equations (24)-(27) determine the state of the particles $x_{i j}(t+1)$ based on the Monte Carlo method:

$$
\begin{gathered}
x_{i j}(t+1)=P_{i j}-\beta \cdot\left(m \text { best }_{j}(t)-x_{i j}(t)\right) \cdot \ln \left(\frac{1}{u_{i j}(t)}\right), \quad \text { for } \rho \geq 0.5 \\
x_{i j}(t+1)=P_{i j}+\beta \cdot\left(m_{\text {best }_{j}}(t)-x_{i j}(t)\right) \cdot \ln \left(\frac{1}{u_{i j}(t)}\right), \quad \text { for } \rho<0.5 \\
P_{i j}(t)=\varphi_{j}(t) \cdot \text { bbest }_{i j}(t)+\left(1-\varphi_{j}(t)\right) \cdot \text { best }_{i j}(t), \\
m \text { best }_{j}(t)=\frac{1}{n} \sum_{i=1}^{n} \text { pbest }_{i j}(t), \\
\beta(t)=\left[\left(\beta_{1}-\beta_{0}\right)(T-t) / T\right]+\beta_{0},
\end{gathered}
$$

where the local best position of each particle is represented by pbest, the global best position of the swarm is represented by gbest, and $m$ best is the mean best position of all individual best positions, pbest. $n$ means the size of the swarm population, $t$ the iteration time-step, $T$ the total number of iterations, $\beta$ is called the contraction-expansion coefficient, and $\beta_{1}$ and $\beta_{0}$ are the upper and lower limits for the coefficient. $\rho, u$, and $\varphi$ are random numbers uniformly distributed between 0 and 1 .

\subsubsection{Multi-objective optimization}

In multiple criteria decision-making, multi-objective optimization is an area that studies the trade-off between conflicting objectives and explores the available design options. Multiobjective optimization problems present multiple responses functions $\boldsymbol{f}\left(\boldsymbol{X}_{i}\right)=\left\{f_{1}\left(\boldsymbol{X}_{i}\right), f_{2}\right.$ $\left.\left(\boldsymbol{X}_{i}\right), \ldots f_{k}\left(\boldsymbol{X}_{i}\right)\right\}^{T}$ for a set of design variables $\boldsymbol{X}_{i}=\left\{X_{i 1}, X_{i 2}, \ldots, X_{i n}\right\}^{T}$. The concept of Pareto front is commonly used to approach multi-objective problems: it is defined that a solution vector $\boldsymbol{f}\left(\boldsymbol{X}_{A}\right)$ dominates another solution vector $\boldsymbol{f}\left(\boldsymbol{X}_{B}\right)$ if, and only if (in case of minimization) $f_{i}\left(\boldsymbol{X}_{A}\right) \leq f_{i}\left(\boldsymbol{X}_{B}\right)$ for $i=1, \ldots, k$ (being $k$ the number of objective functions) and at least one index $j$ holds $f_{j}\left(\boldsymbol{X}_{A}\right)<f_{j}\left(\boldsymbol{X}_{B}\right)$ ). If the mentioned rule is not violated, both design variable vectors are called non-dominated by each other and they form the set $\boldsymbol{P}_{s}$, where $\boldsymbol{P}_{s}=\left\{\boldsymbol{X}_{i} \in \boldsymbol{X} \mid \nexists \boldsymbol{X}_{\boldsymbol{j}} \succ \boldsymbol{X}_{i}\right\}^{T}$, which means that the set of solution vectors $\boldsymbol{X}_{i}$ that belongs to the search space $\boldsymbol{X}$ is such that does not exist a vector $\boldsymbol{X}_{j}$ that dominates $\boldsymbol{X}_{i}$. The set of all objective function vectors that are non-dominated are the so-called Pareto front $\boldsymbol{P}_{f}=\left\{\boldsymbol{f}\left(\boldsymbol{X}_{i}\right)\left|\boldsymbol{X}_{i} \in \boldsymbol{P}_{s}\right|\right\}^{T}$. 


\subsection{The proposed algorithm (MOQPSO)}

The proposed algorithm in this paper is based on the Pareto front archive strategy, similar to that used by Branke and Mostaghim (2006) and Knowles and Corne (1999). The idea behind this strategy is to keep all the best positions stored in a database, where a maintenance routine is performed at the end of each iteration. The archives applied in the proposed algorithm are three, that being:

- $f_{\mathrm{P}}$ archive used to store individual local best positions of each particle;

- $f_{\mathrm{G} 1}$ archive used to store global best positions of the swarm as a whole;

- $f_{\mathrm{G} 2}$ archive used to store global best positions filtered by a distance criterion, thus eliminating clusters of particles;

In this paper, the set of design variables corresponding to each of the above-mentioned archives will be addressed as $\boldsymbol{X}_{\mathrm{G}}, \boldsymbol{X}_{\mathrm{G} 1}$, and $\boldsymbol{X}_{\mathrm{G} 2}$, respectively.

The core equations of quantum particle optimization model described in Eqs. 24-27 are modified accordingly: $m$ best $_{j}(t)$ and $g$ best $_{i j}(t)$ are replaced by guide ${ }_{j}(t)$, and $x_{i j}(t)$ is replaced by $p$ best $_{i j}(t)$. Such modifications applied to the equations are based on an extensive empirical study using 11 multi-objective benchmark functions that includes Schaffer-1, Kursawe, Zitzler-1 to Zitzler-6, Fonseca, Viennet-2, and Viennet-3 (Coello et al. 2007). Summarizing, the classical meanbest variable used in the QPSO is replaced by guide particles in the MOQPSO. These guide particles are chosen and set individually to each new solution created by each particle at every iteration.

The choice of the guide particle has shown to be more effective using a combination of two selected methods: the 'extreme particle' method and the 'nearest particle'. The probability of 'nearest particle' being used instead of 'extreme particle' is previously user-defined as guid_prox.

In the extreme particle case, the number of extreme particles calculated is assumed equal to the number of multi-objective functions. A geometric mean-based choice prevents the algorithm from selecting particles that are too close from each other, increasing the width of the Pareto front. The positions of extreme particles are calculated from archive $f_{\mathrm{G} 1}$ in each iteration, based on the following rules. First, the cumulative distance in objective function space between each particle and other particles is calculated by $d_{i}\left(f_{\mathbf{G}_{1} i}(\boldsymbol{X}), \boldsymbol{f}_{\mathbf{G}_{1} j}(\boldsymbol{X})\right)=$ $\sum_{j=1}^{\mathrm{ng} 1}\left\|\boldsymbol{f}_{\mathbf{G}_{1} i}-\boldsymbol{f}_{\mathbf{G}_{1} j}\right\|_{2}$, for $i=1, \ldots, \mathrm{ng}_{1}$, with $\mathrm{ng}_{1}$ being the number of particles in archive $f_{\mathrm{G} 1}$. Then, the first extreme particle is chosen as:

$$
\begin{gathered}
\boldsymbol{X}_{\mathrm{E} 1}=\underset{\boldsymbol{X} \in \boldsymbol{X}_{\mathrm{G} 1}}{\arg \max } d_{i}\left(\boldsymbol{f}_{\mathbf{G} 1 \boldsymbol{i}}(\boldsymbol{X}), \boldsymbol{f}_{\mathbf{G} 1 \boldsymbol{j}}(\boldsymbol{X})\right) \\
\boldsymbol{f}_{\mathbf{E} 1}\left(\boldsymbol{X}_{\mathrm{E} 1}\right)=\left\{f_{1}\left(\boldsymbol{X}_{\mathrm{E} 1}\right), f_{2}\left(\boldsymbol{X}_{\mathrm{E} 1}\right), \ldots f_{k}\left(\boldsymbol{X}_{\mathrm{E} 1)\}^{T} .}\right.\right.
\end{gathered}
$$

The second extreme particle is chosen based on the previous extreme particle as:

$$
\begin{gathered}
\boldsymbol{X}_{\mathrm{E} 2}=\underset{\boldsymbol{X} \in \boldsymbol{X}_{\mathrm{G} 1}}{\arg \max } \boldsymbol{f}_{\mathrm{E} 1}\left(\boldsymbol{X}_{\mathrm{E} 1}\right)-\boldsymbol{f}_{\boldsymbol{G} 1 i_{2}} \text { for } i=1, \ldots, \mathrm{ng} 1-1 \\
\boldsymbol{f}_{\mathrm{E} 2}\left(\boldsymbol{X}_{\mathrm{E} 2}\right)=\left\{f_{1}\left(\boldsymbol{X}_{\mathrm{E} 2}\right), f_{2}\left(\boldsymbol{X}_{\mathrm{E} 2}\right), \ldots f_{k}\left(\boldsymbol{X}_{\mathrm{E} 2}\right)\right\}^{T} .
\end{gathered}
$$

For the third extreme, a geometric mean is used and evaluated as $d g_{i}$ $\left(f_{\mathrm{E} 1}\left(X_{\mathrm{E} 1}\right), f_{\mathrm{E} 2}\left(X_{\mathrm{E} 2}\right), f_{\mathrm{G} 1 j}(X)\right)=f_{\mathrm{E} 1}\left(X_{\mathrm{E} 1}\right)-f_{\mathrm{G} 1 i_{2}} f_{\mathrm{E} 2}\left(X_{\mathrm{E} 2}\right)-f_{\mathrm{G} 1 i_{2}}$, for $i=$ $1, \ldots, \mathrm{ng}_{1}-2$, and the extreme particle is defined as:

$$
\begin{gathered}
\boldsymbol{X}_{\mathrm{E} 3}=\underset{\boldsymbol{X} \in \boldsymbol{X}_{\mathrm{G} 1}}{\arg \max } d g_{i}\left(\boldsymbol{f}_{\mathrm{E} 1}\left(\boldsymbol{X}_{\mathrm{E} 1}\right), \boldsymbol{f}_{\mathrm{E} 2}\left(\boldsymbol{X}_{\mathrm{E} 2}\right), \boldsymbol{f}_{\mathbf{G} 1 \boldsymbol{j}}(\boldsymbol{X})\right) \\
\boldsymbol{f}_{\mathrm{E} 3}\left(\boldsymbol{X}_{\mathrm{E} 3}\right)=\left\{f_{1}\left(\boldsymbol{X}_{\mathrm{E} 3}\right), f_{2}\left(\boldsymbol{X}_{\mathrm{E} 3}\right), \ldots f_{k}\left(\boldsymbol{X}_{\mathrm{E} 3}\right)\right\}^{T} .
\end{gathered}
$$




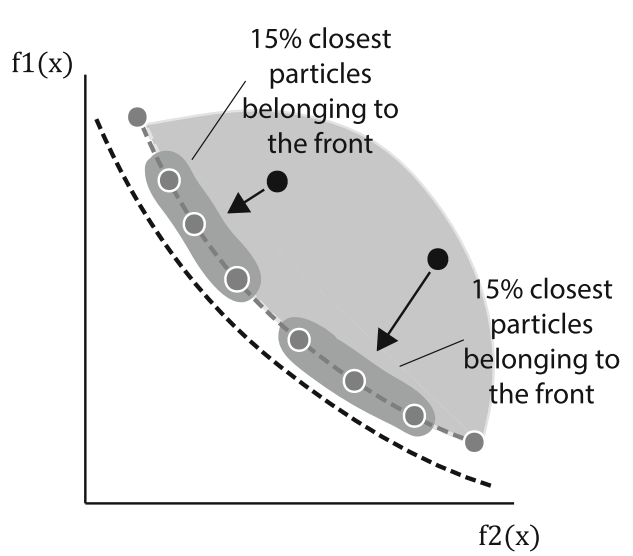

(a)

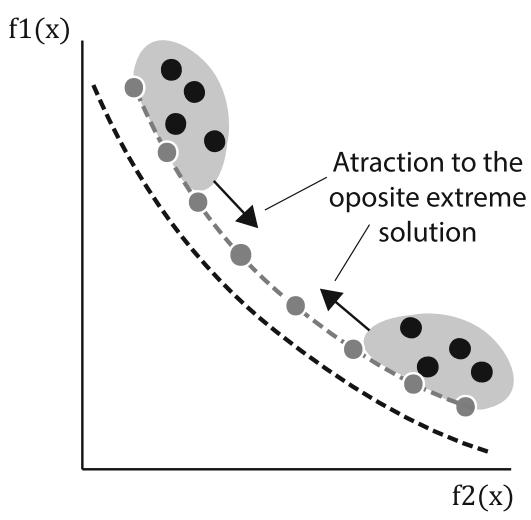

(b)

Fig. 4 a Behavior induced by the extreme guide mechanism. b Behavior induced by nearest particles belonging to the Pareto front guide mechanism

As a general rule, for the 3rd extreme onwards, an extreme $e$ can be evaluated based on the same reasoning, $d g_{i}=\prod_{l=1}^{e-1}\left\|\boldsymbol{f}_{\mathrm{El}}\left(\boldsymbol{X}_{\mathrm{El}}\right)-\boldsymbol{f}_{\mathrm{G} 1 i}\right\|_{2}$, for $i=1, \ldots, \mathrm{ng}_{1}-(e-1)$, so the extreme particle is defined as:

$$
\begin{gathered}
\boldsymbol{X}_{\mathrm{Ee}}=\underset{\boldsymbol{X} \in \boldsymbol{X}_{\mathrm{G} 1}}{\arg \max } d g_{i}, \\
\boldsymbol{f}_{\mathrm{E} 3}\left(\boldsymbol{X}_{\mathrm{E} 3}\right)=\left\{f_{1}\left(\boldsymbol{X}_{\mathrm{E} 3}\right), f_{2}\left(\boldsymbol{X}_{\mathrm{E} 3}\right), \ldots f_{k}\left(\boldsymbol{X}_{\mathrm{E} 3}\right)\right\}^{T} .
\end{gathered}
$$

The algorithm then chooses the farthest extreme particle. This type of selection induces the behavior described in Fig. 4a, in the case of two objective functions, that produces a wide Pareto front and increases the spread among particles. It was observed that the implementation of the geometric-based approach increased, at the same time, the algorithm's convergence and spread when tested with some of the benchmark functions aforementioned, which is a remarkable trait for a multi-objective mechanism and one of the innovations brought by the present work.

Figure $4 \mathrm{~b}$ describes the behavior induced by the guide particle when the selection is done by the nearest particles belonging to the Pareto front. This mechanism increases the convergence of the algorithm. The selection is smoothed by choosing randomly the guide particle among the list of $n$th nearest particles to maintain diversity. The number of $n$th is assumed $15 \%$ of the $f_{\mathrm{G} 1}$ archive (from now on defined guid_perc). As for constraint handling, if an infeasible solution $\mathbf{x}$ is obtained when generating the particles, it is discarded and a new solution is generated to replace it.

A simplified flowchart of the MOQPSO algorithm is depicted in Fig. 5.

\section{Numerical simulations: half-car suspension problem}

In this section, a half-car vertical dynamics suspension model is investigated as described in Boonlong (2013). The vehicle moves at a constant speed of $20 \mathrm{~m} / \mathrm{s}$ and is excited by a sinusoidal double road bump with an amplitude of $0.05 \mathrm{~m}$. Similar to Boonlong (2013), in this paper, five objective functions were taken in the study. Output values like driver seat 


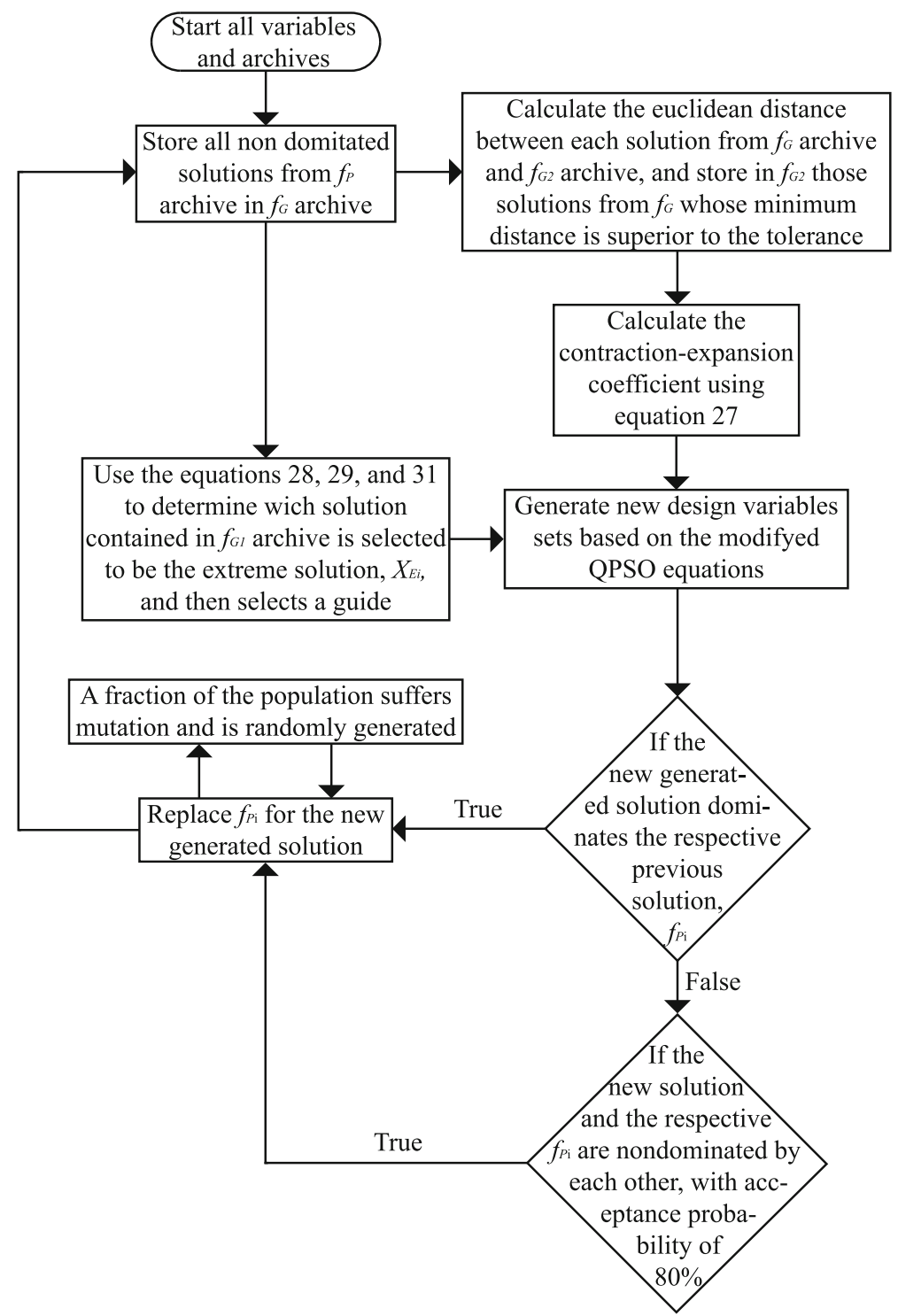

Fig. 5 Simplified flowchart of the proposed MOQPSO algorithm

acceleration $\left(x_{1}\right)$, front tire velocity $\left(x_{2}\right)$, rear tire velocity $\left(x_{3}\right)$, front suspension working space relative displacement between sprung mass and front tire, $\left(x_{4}\right)$, and rear suspension working space $\left(x_{5}\right)$ were used as part of the objective functions. For comparisons purposes, the values for the objective functions were taken as indicated by Boonlong (2013) as:

$$
f_{i}=\left[\int_{0}^{T} x_{i}^{2} \mathrm{~d} t\right]^{1 / 2}, \quad i=1,2, \ldots, 5
$$




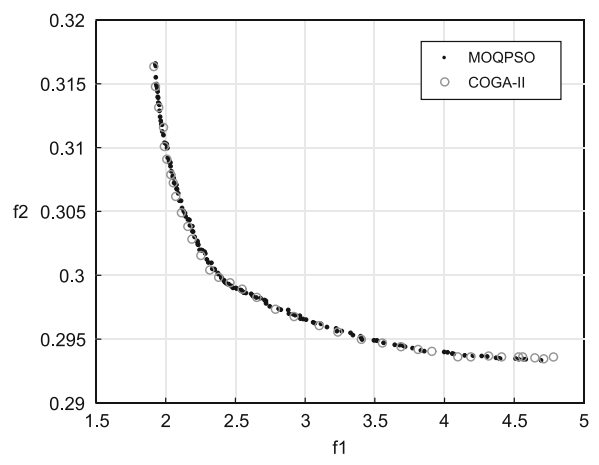

(a)

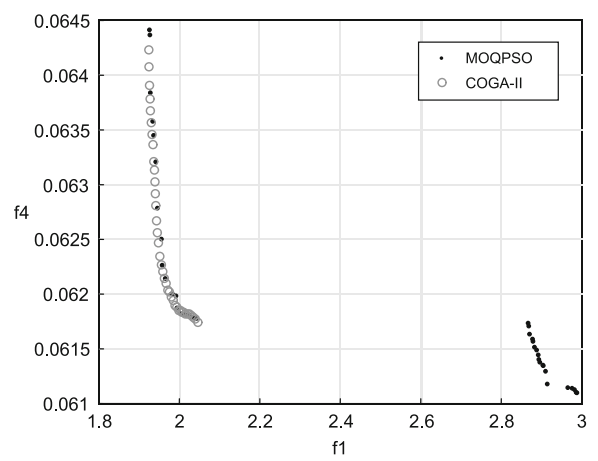

(c)

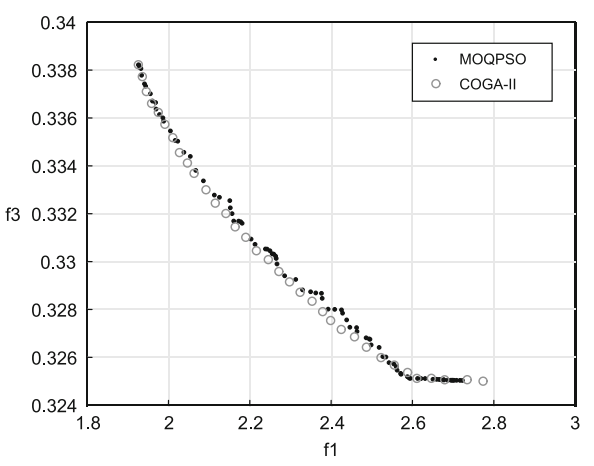

(b)

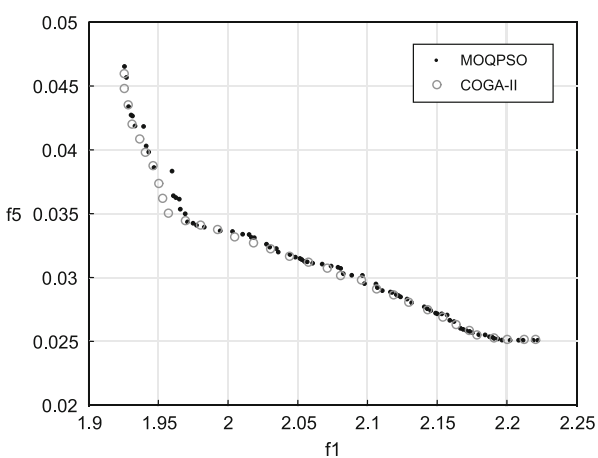

(d)

Fig. 6 Resulted Pareto front using MOQPSO and COGA-II. a $f_{1} \times f_{2}$, b $f_{1} \times f_{3}, \mathbf{c} f_{1} \times f_{4}$, and $\mathbf{d} f_{1} \times f_{5}$

Equation (32) resembles the RMS value, but did not comply with the correct form, since it should be instead $f_{i}=\left[(1 / T) \int_{0}^{T} x_{i}^{2} \mathrm{~d} t\right]^{1 / 2}$ where, in this case, the units for $f_{i}$ will be the same as $x_{i}$. The original Boonlong (2013) is suggested for the reader who wants more detailed information about this example. It is important to notice that this suspension model is very simple and can only deal with pitch and heave and cannot address other important degrees of freedom like roll, yaw, surge, and sway that may interact with each other resulting in complex dynamic behaviors.

To perform the multi-objective optimization, groups of two objective functions were set in a bi-objective optimization process $\left(f_{1} \times f_{2}, f_{1} \times f_{3}, f_{1} \times f_{4}, f_{1} \times f_{5}\right)$. In the end, all five objective functions are taken together in multi-objective optimization $\left(f_{1} \times f_{2} \times f_{3} \times f_{4} \times f_{5}\right)$. Seven design variables were used for the optimizations. The stiffness $\left(k_{\mathrm{ss}}\right)$ and damping $\left(c_{\mathrm{ss}}\right)$ of the driver seat, the stiffness $\left(k_{\mathrm{sf}}\right)$ and damping $\left(c_{\mathrm{sf}}\right)$ of the front wheel, the stiffness $\left(k_{\mathrm{sr}}\right)$ and damping $\left(c_{\mathrm{sr}}\right)$ of the rear wheel, and the position of the driver seat $(r)$ relative to the half-car center of mass (CM).

Figure 6 shows the bi-objective Pareto fronts for the objective function combinations $f_{1} \times f_{2}, f_{1} \times f_{3}, f_{1} \times f_{4}, f_{1} \times f_{5}$. In general, the results obtained by MOQPSO are similar to those presented by Boonlong (2013). However, as one can see in Fig. 6c, the MOQPSO found a new region on the Pareto front that is far from that presented by Boonlong (2013). This shows that the proposed algorithm has scattering and exploring features. In all bi-objective 

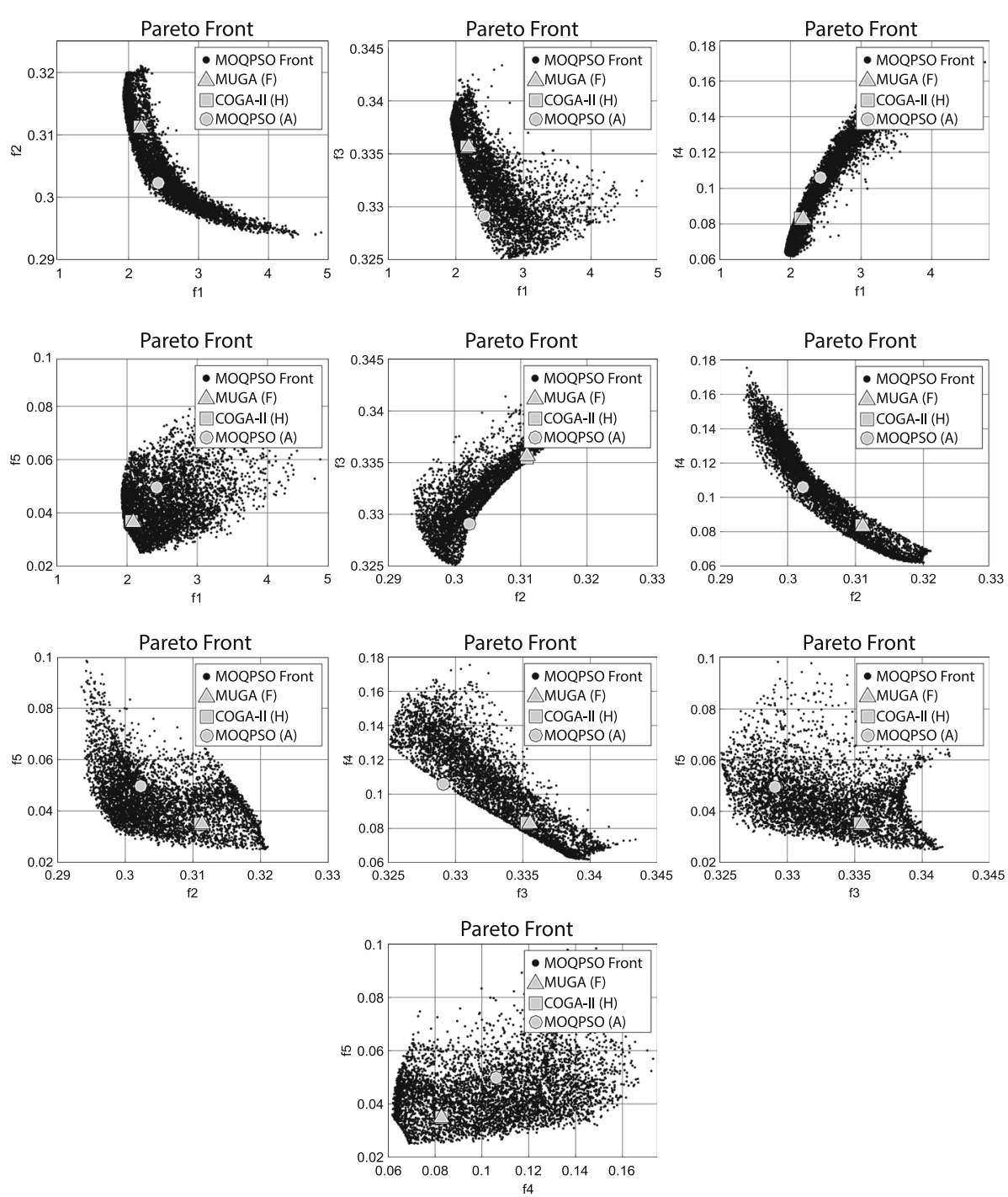

Fig. 7 Projected views of multi-objective Pareto fronts for the half-car problem

analysis, 800 iterations with 60 individuals were used and tolerance of tol $=1 \times 10^{-3}$ is used as a limit for the point to be included in the Pareto front archive. An average number of 53,720 function evaluations were performed.

As the last example, all five objective functions were optimized at the same time. The results are presented in Fig. 7. Notice that the result presented by Boonlong (2013) is encompassed by the Pareto front found in this work in all projected views, which means that those solutions are covered in the MOQPSO's Pareto front.

Table 2 shows the objective function and design variable values for Point $F$ (MUGA), Point $H$ (COGA-II) and the result obtained by MOQPSO called point A. For comparison purposes only, the sum of normalized values of each objective function is used, i.e., $B=$ 
Table 2 Best design variables/objective functions for the five multi-objective optimization cases

\begin{tabular}{llll}
\hline & MUGA $(\mathrm{F})^{\mathrm{a}}$ & COGA-II $(\mathrm{H})^{\mathrm{a}}$ & MOQPSO (A) \\
\hline$k_{\mathrm{SS}}$ & 144902 & 50115 & 50000 \\
$c_{\mathrm{SS}}$ & 2788.2 & 4000 & 3886.8 \\
$k_{\mathrm{Sf}}$ & 10000 & 10000 & 10000 \\
$c_{\mathrm{Sf}}$ & 1294.1 & 1290 & 786.51 \\
$k_{\mathrm{Sr}}$ & 10196 & 10034 & 10000 \\
$c_{\mathrm{Sr}}$ & 1982.4 & 1999.8 & 1137.8 \\
$r$ & 0.49608 & 0.49986 & 0.5 \\
$f_{1}$ & 2.185731 & 2.158443 & $\mathbf{2 . 4 2 5 4 0 0}$ \\
$f_{2}$ & 0.311041 & 0.310966 & $\mathbf{0 . 3 0 2 2 9 0 0}$ \\
$f_{3}$ & 0.335592 & 0.335391 & $\mathbf{0 . 3 2 9 1 1 0 0}$ \\
$f_{4}$ & 0.082842 & 0.082632 & $\mathbf{0 . 1 0 6 0 6 0 0}$ \\
$f_{5}$ & 0.034964 & 0.034810 & $\mathbf{0 . 0 4 9 5 8 7 0 0}$ \\
$B$ & 1.6216 & 1.5942 & $\mathbf{1 . 4 3 6 5}$ \\
\hline
\end{tabular}

${ }^{\mathrm{a}}$ Ref. Boonlong (2013)
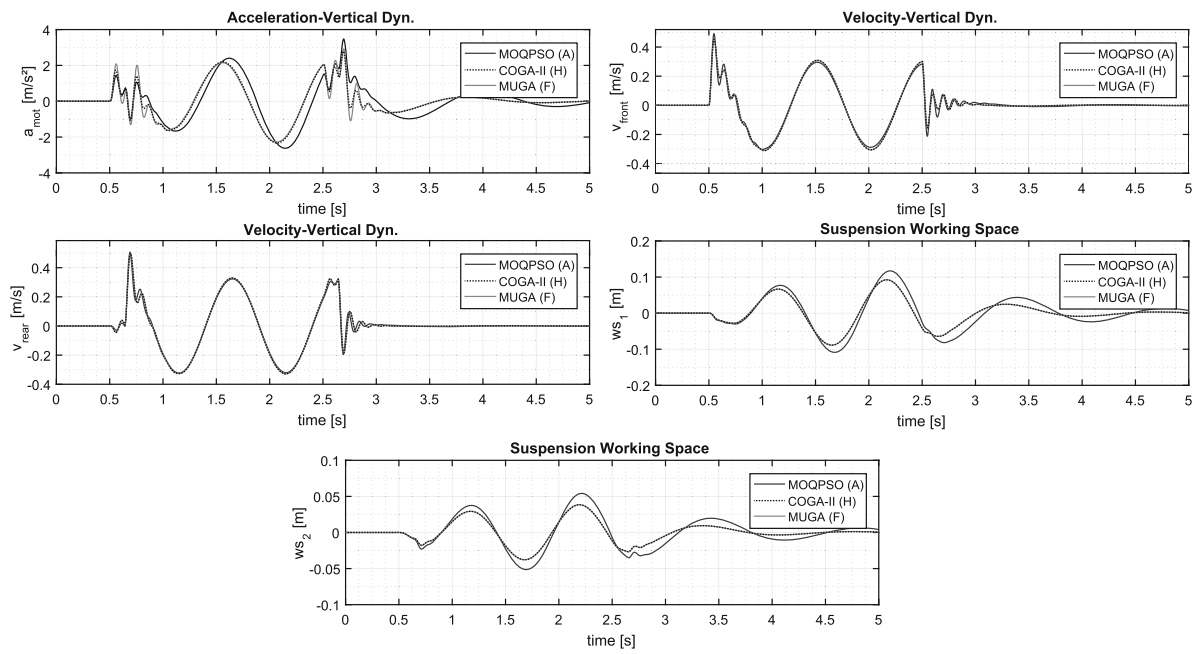

Fig. 8 Time history for $x_{1}, x_{2}, x_{3}, x_{4}$ and $x_{5}$ output parameters for the five objective function optimization in comparison to that found by (Boonlong 2013)

$\min _{j}\left\{\sum_{i=1}^{5}\left[f_{i, j}-\min \left(f_{i}\right)\right] /\left[\max \left(f_{i}\right)-\min \left(f_{i}\right)\right]\right\}$, where $f_{i, j}$ is the $i$ th objective function value for solution $j$, and $f_{i}$ are the $i$ th objective function value for all solutions in the Pareto front. Table 2 shows that the proposed algorithm presented a better $B$ value for the obtained solution in this case.

Figure 8 shows the time history of the objective function $x_{1}$ for the best solution found in this paper in comparison with the best solution found by Boonlong (2013) for the simultaneous five objective optimization case.

The best solution found by MOQPSO showed better results for functions $f_{2}$ and $f_{3}$ (front and rear tires vertical velocity) when compared to Boonlong (2013) the best solution. This behavior results from the lower damping in the front and rear parts of the suspension. However, 
the low damping parameter increases the working space and the driver's acceleration, as can be seen in Fig. 8. The vertical velocity of front wheel shows some higher peaks, but in general lower values in MOQPSO solution than in Boonlong (2013) solution. As for the rear velocity, the solution found in the paper shows lower peaks. The optimum found showed some higher acceleration peaks and other lower acceleration of the driver's seat (Fig. 8). In general, the oscillatory behavior showed to be less damped. The comparison criterion $B$ shows that the proposed algorithm presented a lower $B$ value in the half-car example, meaning that MOQPSO's point $A$ is, in general, a better option when accounting for all five objective functions simultaneously. It is worth to mention these conclusions are limited to the simple half-car suspension model that allows dealing with pitch and heave and may not completely resemble the actual dynamics of a complete vehicle suspension model. To improve the vehicle model, the lateral dynamics from vehicle maneuvers and the road irregularities will be included in the next example with a more elaborated bus suspension model.

\section{Numerical simulations: bus suspension problem}

\subsection{Definition of the multi-objective problem}

The multi-objective problem is the minimization of the vertical root-mean-square acceleration for the driver and two passengers simultaneously, hence the multi-objective approach. The position of the passengers in the bus is depicted in Fig. 2, and the multi-objective problem is described as follows:

$$
\begin{array}{cl}
\text { Minimize }: & f_{i}(x)=a_{z \mathrm{rms}, i}, \quad i=1,2,3 \\
\text { Subject to : } & \left|w_{s j}\right|<w_{s 0 j}, \quad j=1,2,3,4 \\
& F_{z j} \geq 0, \quad j=1,2,3,4 \\
& x_{i} \in\left[0,75 \cdot x_{i, \mathrm{ref}} ; 1,25 \cdot x_{i, \mathrm{ref}}\right]
\end{array}
$$

where $f_{i}(x)$ are the multiple objective functions, $x_{i}$ are the design variables, $w_{s j}$ is the working space in each suspension, $w_{s 0 j}$ is the initial settlement for each suspension and to assure road-holding in the simulation, and the reaction forces in each tire $\left(F_{z j}\right)$ are checked against negative values. The geometric and inertial parameters, as well as the stiffness and damping parameters used in the bus numeric simulation, are listed in Tables 3 and 4, respectively.

The selected design variables in the optimization are $k_{\mathrm{sv}}, k_{\mathrm{sp} 1}, k_{\mathrm{sp} 2}, k_{\mathrm{p}}, k_{\mathrm{z}}, c_{\mathrm{sv}}, c_{\mathrm{sp} 1}, c_{\mathrm{sp} 2}$, $c_{\mathrm{p}}, c_{\mathrm{z}}$ which are parameters that can be easily modified in the design phase of a vehicle suspension. All of the design variables are bounded to an interval of $\pm 25 \%$ of the reference values shown in Table 4. Because the passenger's seats are considered the same in the entire bus, the parameters $k_{\mathrm{sp} 1}$ and $k_{\mathrm{sp} 2}$, as well as parameters $c_{\mathrm{sp} 1}$ and $c_{\mathrm{sp} 2}$, are assumed equal, meaning that a number of eight design variables were used in the optimization.

In this example, the bus numerical model is set to travel at $80 \mathrm{~km} / \mathrm{h}$ on a road class $\mathrm{C}$ (ISO 8608 1995) and perform the DLC (ISO 3888-2 1999). The track was proportionally stretched from the standard dimension by 4.8 times, to make the maneuver feasible by the vehicle. In addition to the road profile, this maneuver will be the main source for lateral vibration excitation. The required time for each deterministic simulation took about $1.3 \mathrm{~s}$ using a computer with $2.6 \mathrm{GHz}$ and $8 \mathrm{~GB}$ of RAM. 
Table 3 Geometric and inertial parameters used in the bus simulation Adapted from Sekulic et al. (2013)

\begin{tabular}{|c|c|c|}
\hline Variables & Geometric and inertial parameters & Nominal value \\
\hline$m$ & Fully loaded bus elastic-suspended mass & $15,890 \mathrm{~kg}$ \\
\hline$m_{\mathrm{p} 1}$ & Passenger 1 and seat mass & $90 \mathrm{~kg}$ \\
\hline$m_{\mathrm{p} 2}$ & Passenger 2 and seat mass & $90 \mathrm{~kg}$ \\
\hline$m_{\mathrm{V}}$ & Driver and seat mass & $100 \mathrm{~kg}$ \\
\hline$m_{\mathrm{pm}}$ & Mass of the front axle & $746 \mathrm{~kg}$ \\
\hline$m_{\mathrm{zm}}$ & Mass of the rear axle & $1355 \mathrm{~kg}$ \\
\hline$J_{\mathrm{x} 1}$ & Moment of inertia of the front axle relative to its centerline & $350 \mathrm{~kg} \mathrm{~m}^{2}$ \\
\hline$J_{\mathrm{x} 2}$ & Moment of inertia of the rear axle relative to its centerline & $620 \mathrm{~kg} \mathrm{~m}^{2}$ \\
\hline$J_{x}$ & $x$-axis suspended mass moment of inertia & $13,000 \mathrm{~kg} \mathrm{~m}^{2}$ \\
\hline$J_{y}$ & $y$-axis suspended mass moment of inertia & $150,000 \mathrm{~kg} \mathrm{~m}^{2}$ \\
\hline$J_{z}$ & The suspended mass moment of inertia relative to the $z$-axis & $195,980 \mathrm{~kg} \mathrm{~m}^{2}$ \\
\hline$a$ & Distance between the front axle and bus CM & $3.61 \mathrm{~m}$ \\
\hline$b$ & Distance between the rear axle and bus CM & $2.04 \mathrm{~m}$ \\
\hline$l$ & Wheelbase & $5.65 \mathrm{~m}$ \\
\hline$e_{1}$ & Distance between suspension elements on the front axle and front axle CM & $0.70 \mathrm{~m}$ \\
\hline$e_{2}$ & Distance between suspension elements on the rear axle and rear axle CM & $0.80 \mathrm{~m}$ \\
\hline$f_{1}$ & Distance between front-right and left wheels and front CM & $1.00 \mathrm{~m}$ \\
\hline$f_{2}$ & Distance between rear right and left wheel and rear axle CM & $1.00 \mathrm{~m}$ \\
\hline$S_{1}$ & Distance between the driver seat and the longitudinal $x$-axis & $0.65 \mathrm{~m}$ \\
\hline$S_{2}$ & Distance between the driver seat and the transversal $y$-axis & $5.45 \mathrm{~m}$ \\
\hline$S_{3}$ & Distance between passenger 1 seat and the longitudinal $x$-axis & $0.80 \mathrm{~m}$ \\
\hline$S_{4}$ & Distance between passenger 1 seat and the transversal $y$-axis & $0.50 \mathrm{~m}$ \\
\hline$S_{5}$ & Distance between passenger 2 seat and the longitudinal $x$-axis & $0.40 \mathrm{~m}$ \\
\hline$S_{6}$ & Distance between passenger 2 seat and the transversal $y$-axis & $4.20 \mathrm{~m}$ \\
\hline
\end{tabular}

\subsection{Optimization parameters}

The following parameters were used for the MOQPSO: number of particles $(n=30)$; tolerance limit (tol1 $=0.001)$; contraction expansion initial limit $\left(\beta_{0}=1.3\right)$; contraction expansion final limit $\left(\beta_{1}=0.3\right.$ ); the percentage of the nearest individuals belonging to the Pareto front to be drawn as guide (guid_perc $=0.15$ ); the probability of 'closest guide' being used instead of the extreme guide ( guid_prox $=0.30$ ); mutation probability (mutation $=0.12$ ). All parameters were tested with different values during the calibration phase. In the final choice of these parameters, as a rule of thumb, it was considered that an excessive increase in the mutation value, decrease in guid_prox, and/or increase in guid_perc, impairs the convergence rate. Increasing guid_prox created a short Pareto front. Significant modifications in $\beta_{0}$ and $\beta_{1}$ implied in lack of convergence or lack of diversity.

For the NSGA-II algorithm, the following parameters were used: crossover fraction (cr $=0.8)$; Pareto fraction ( $\mathrm{pf}=0.9)$; population number $(n=800)$; (distancecrowding $=$ 'phenotype'); once again, all parameters were tested with different values during the calibration phase. Populations between 10 and 1000 were tested, where 800 showed to be the best value for the specific bus problem. Low values for population numbers would result in a short 
Table 4 Stiffness and damping parameters used in the bus simulation Adapted from Sekulic et al. (2013)

\begin{tabular}{lll}
\hline Variables & Suspension parameters & Nominal value \\
\hline$k_{\mathrm{Sv}}$ & Driver seat spring stiffness & $10,000 \mathrm{~N} / \mathrm{m}$ \\
$c_{\mathrm{sV}}$ & Driver seat shock-absorber damping & $750 \mathrm{Ns} / \mathrm{m}$ \\
$k_{\mathrm{sp} 1}, k_{\mathrm{sp} 2}$ & Passenger 1 and passenger 2 seat stiffness & $40,000 \mathrm{~N} / \mathrm{m}$ \\
$c_{\mathrm{sp} 1}, c_{\mathrm{sp} 2}$ & Passenger 1 and passenger 2 seat damping & $220 \mathrm{Ns} / \mathrm{m}$ \\
$k_{\mathrm{p}}$ & Front axle single airbag stiffness & $175,000 \mathrm{~N} / \mathrm{m}$ \\
$c_{\mathrm{p}}$ & Left and the right side equivalent shock-absorber damping of the front axle & $40,000 \mathrm{Ns} / \mathrm{m}$ \\
$c_{1}$ & Front axle single shock-absorber stiffness & $20,000 \mathrm{Ns} / \mathrm{m}$ \\
$k_{2}$ & Rear axle single airbag stiffness & $200,000 \mathrm{~N} / \mathrm{m}$ \\
$c_{2}$ & Rear axle single shock-absorber damping & $22,500 \mathrm{Ns} / \mathrm{m}$ \\
$k_{\mathrm{Z}}$ & Left and the right side equivalent airbags stiffness of the rear axle & $408,650 \mathrm{~N} / \mathrm{m}$ \\
$c_{\mathrm{Z}}$ & Left and the right side equivalent shock-absorber damping of the rear axle & $45,973 \mathrm{Ns} / \mathrm{m}$ \\
$k_{\mathrm{pp}}$ & Rear axle single tire stiffness on the front & $1,000,000 \mathrm{~N} / \mathrm{m}$ \\
$c_{\mathrm{pp}}$ & Front and rear axle single tire damping & $150 \mathrm{Ns} / \mathrm{m}$ \\
$k_{\mathrm{zp}}$ & Left and the right side equivalent tire stiffness of the rear axle & $2,000,000 \mathrm{~N} / \mathrm{m}$ \\
$c_{\mathrm{zp}}$ & Left and right side of the rear axle equivalent tire damping & $300 \mathrm{Ns} / \mathrm{m}$ \\
\hline
\end{tabular}

and insufficient front, and high numbers would significantly impair convergence. As for the other parameters, decreasing Pareto fraction or increasing crossover fraction would make the front wider at the cost of convergence. The inverse was also true, where increasing Pareto fraction or decreasing crossover fraction would make the front shorter, but slightly increase convergence. The distance crowding parameter set as 'phenotype' showed an overall better result when compared to 'genotype'.

\subsection{Optimization results, comparisons, and discussion}

All the following result comparisons are based on the numeric bus model traveling at a constant speed of $80 \mathrm{~km} / \mathrm{h}$ on a road class C (ISO 8608 1995) and performing the DLC test (ISO 3888-2 1999). Since this model presents complex behavior and there is no equivalent experiment in the literature, the results will be compared with a well-known multi-objective algorithm NSGA-II. Therefore, the results from the novel multi-objective algorithm can be compared to those from NSGA-II in Fig. 9 (projected views of the Pareto front), where the number of simulations for each algorithm was 135,324 . The Pareto front obtained by NSGAII is completely dominated by that obtained using MOQPSO. Besides, it is possible to notice that the MOQPSO Pareto front is wider and more spread, reaching places unexplored by NSGA-II.

Notice that the problem holds three objective functions, meaning that clusters observed in Fig. 9 may actually be spread in the three-dimensional space. Comparisons between specific points in the Pareto front obtained with MOQPSO and NSGA-II are meaningless, since the range of NSGA-II solutions did not reach the extension obtained by the MOQPSO.

A solution using an equally weighted monobjective function and a simple QPSO algorithm was obtained, and it was observed that it agrees with the nearest point to the origin of the previous Pareto front. As expected, this approach is a particular solution from the Pareto front. This equally weighted monobjective solution was slightly ahead of the Pareto front, 


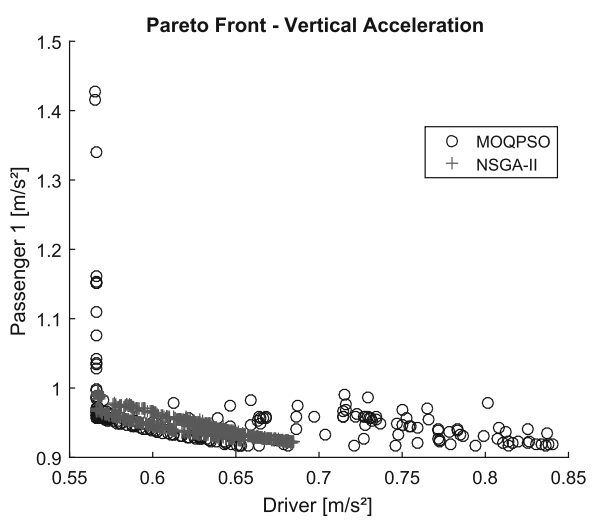

(a)

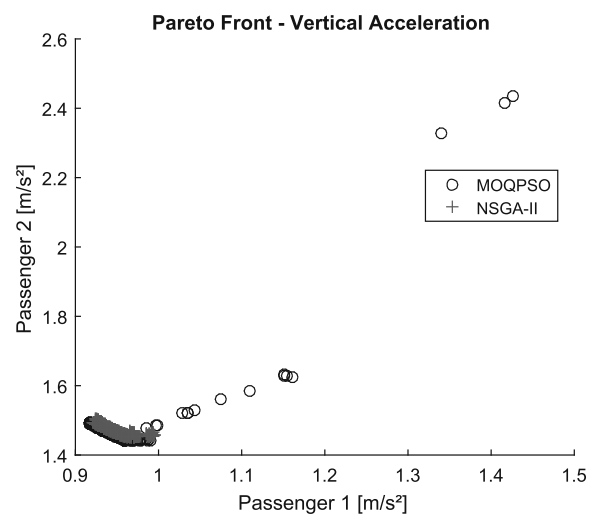

(c)

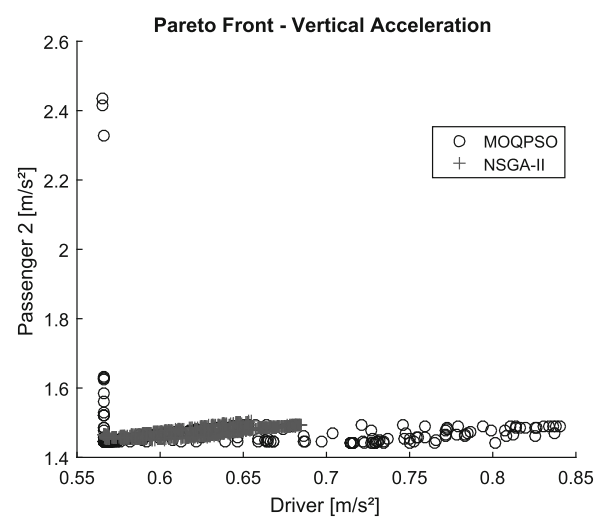

(b)

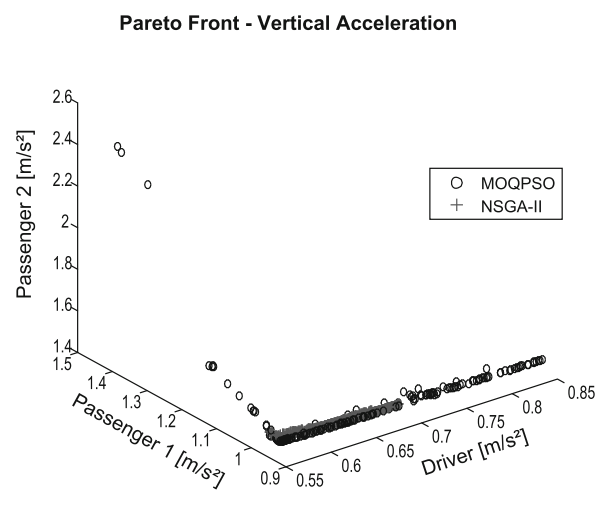

(d)

Fig. 9 Resulted Pareto front using NSGA-II and MOQPSO. a Passenger 1 versus passenger 2 view, b driver versus passenger 1 view, $\mathbf{c}$ driver versus passenger 2 view, and $\mathbf{d}$ perspective view

indicating that the Pareto front would be further improved at the cost of a higher computational time.

Different views of the resulting Pareto front using MOQPSO set for 291,932 simulations are presented in Fig. 10. Table 5 shows the corresponding design variables for each of the solution points in the Pareto front marked with circles and labeled with capital letters. Point $H$ holds the solution for the nominal parameter values.

From Table 5, it is noteworthy that passenger 1 and 2 seat stiffness, $k_{\mathrm{sp} 1}$ and $k_{\mathrm{sp} 2}$, and the equivalent airbags stiffness on the left and the right side of the rear axle $k_{z}$ are kept to the lower bound (that being $75 \%$ of the nominal values). Previous works such as Sharp and Crolla (1987) have shown that lower stiffness and damping reduces the acceleration upon the car body, in a quarter-car suspension model. This result is confirmed in the presented more complex model.

Nevertheless, the single airbag stiffness on the front axle $\left(k_{\mathrm{p}}\right)$ and the spring stiffness of the driver seat $\left(k_{\mathrm{sv}}\right)$ are not kept to a minimum in all solutions, which is an apparent contradiction to what was said previously. Figure 10 and Table 5 shows that solutions $E, F$, and $G$ result in a decrease in the passengers' vertical acceleration while increasing the driver's acceleration. 


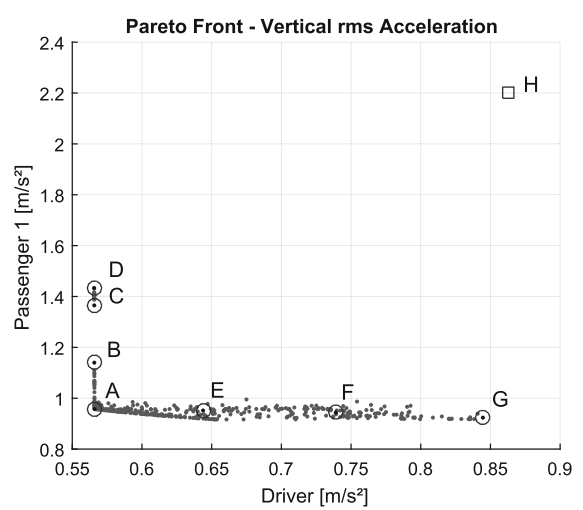

(a)

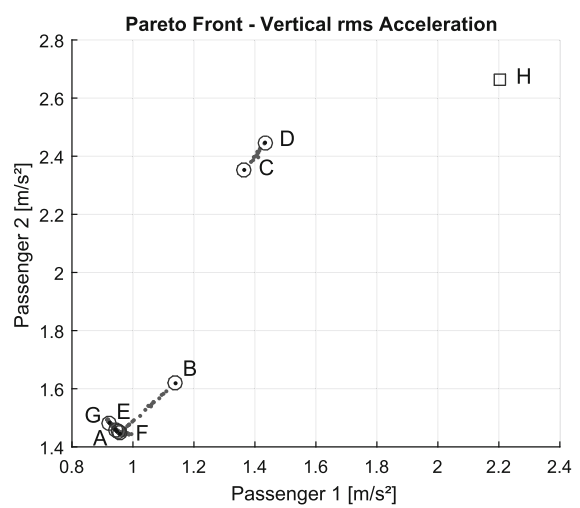

(c)

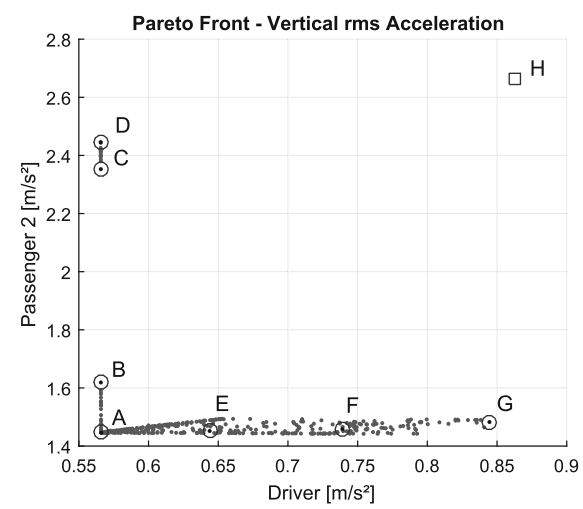

(b)

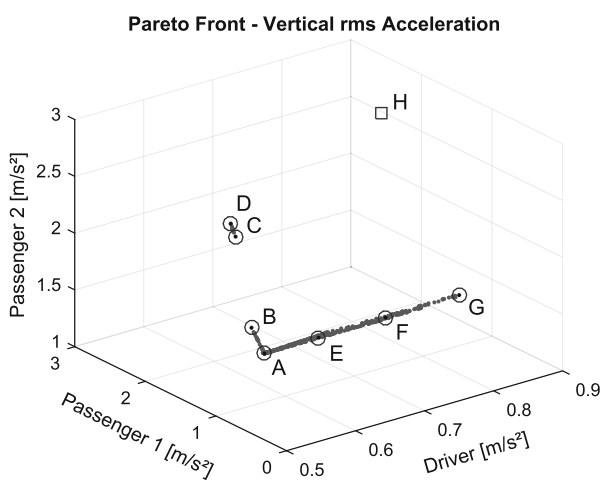

(d)

Fig. 10 Resulted Pareto front using MOQPSO. a Passenger 1 versus passenger 2 view, $\mathbf{b}$ driver versus passenger 1 view, c driver versus passenger 2 view, and (d) perspective view

One hypothesis to explain this behavior is that the harder the single airbag stiffness on the front axle $\left(k_{\mathrm{p}}\right)$ and the spring stiffness of the driver seat $\left(k_{\mathrm{sv}}\right)$ are, the smaller the relative displacement between sprung mass and suspension is. This might lead to an increase in the acceleration at the front of the vehicle. On the other hand, the rest of the suspension is prone to dissipate more energy, which leads to lower accelerations for passengers 1 and 2. This behavior was also checked based on the dissipated energy evaluations on front and rear axle dampers, where more energy is locally dissipated at a higher relative speed with small damping values.

The equivalent shock-absorber damping on the left and the right side of the front axle $\left(c_{\mathrm{p}}\right)$ is also kept to the lower bound, while its rear counterpart $\left(c_{\mathrm{z}}\right)$ follows the same pattern with the exception of solutions $C$ and $D$ (see Fig. 10 and Table 5). This is an interesting result where a slight decrease in the driver's acceleration compromises the passengers' acceleration. In cases $C$ and $D$, all the design variables are kept constant, except for $c_{\mathrm{sv}}, c_{\mathrm{sp} 1}, c_{\mathrm{sp} 2}$, and $c_{\mathrm{z}}$. As expected, when the last design variable, equivalent shock-absorber damping on the left and the right side of the rear axle, $\left(c_{\mathrm{z}}\right)$ increases, the passengers' acceleration also increases. The reason behind the decrease in the passengers' accelerations may be explained by the previous hypothesis analogously. 


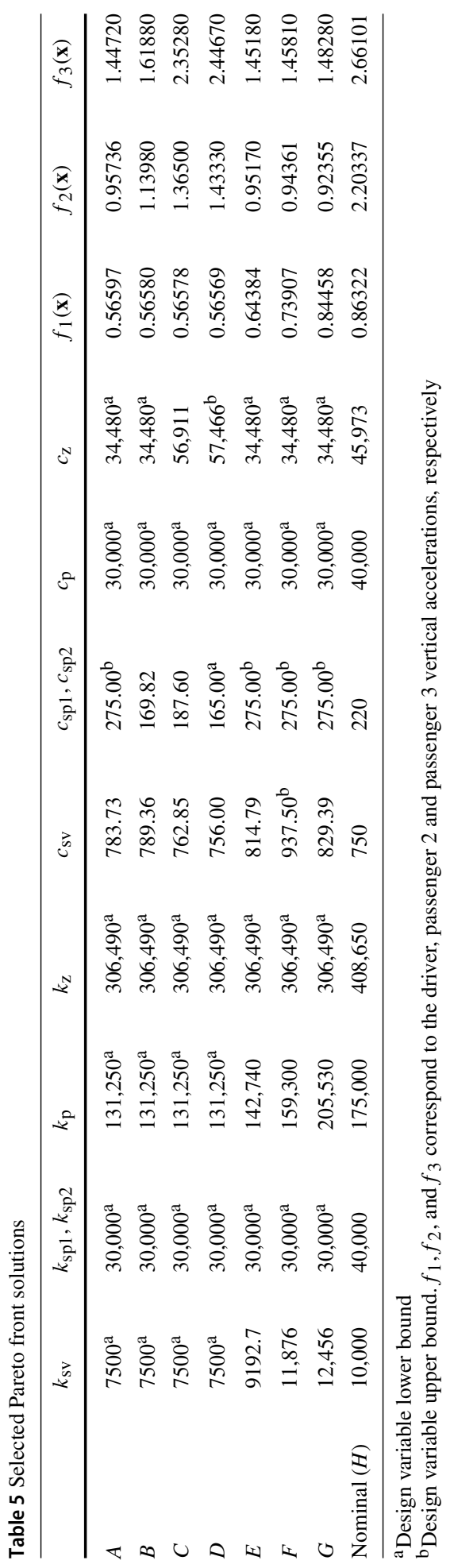


Table 6 Maximum absolute suspension working space at DLC maneuvering

\begin{tabular}{lllllllll}
\hline Pareto front case & $A$ & $B$ & $C$ & $D$ & $E$ & $F$ & $G$ & Nominal $H$ \\
\hline $\begin{array}{c}\text { Suspension working } \\
\text { space (m) }\end{array}$ & 0.407 & 0.407 & 0.411 & 0.412 & 0.411 & 0.412 & 0.418 & 0.389 \\
\hline
\end{tabular}
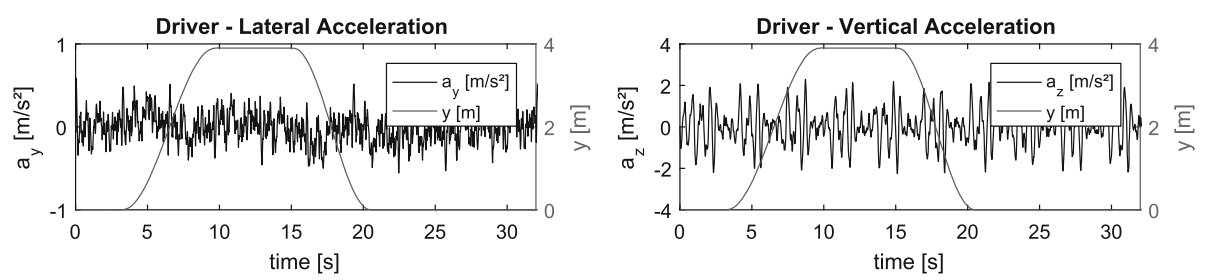

(a)
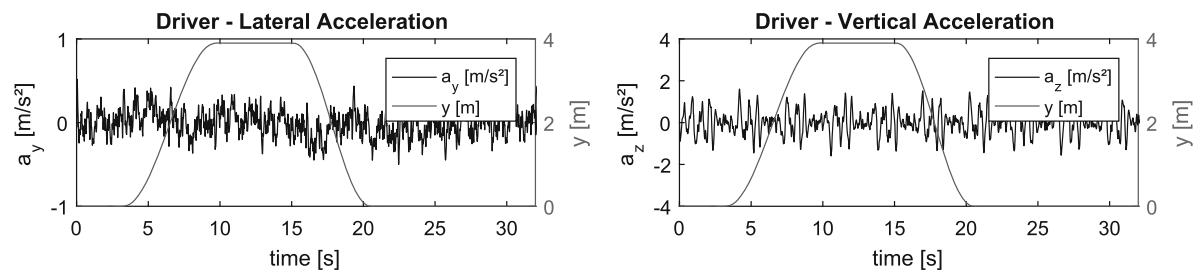

(b)

Fig. 11 Typical lateral and vertical acceleration signals for the driver: a nominal solution, $H$; b optimized solution, $A$ (lateral displacement of bus $C G$ at right $y$-axis for reference)

Although its well known that lower stiffness and damping reduces the vertical RMS acceleration upon the passengers (Sharp and Crolla 1987), the behavior of solutions in the present study shows reduction in acceleration on different places of the bus model by increasing stiffness or damping in opposite places of the suspension (see solutions $C$ and $D$, Table 5). This result indicates that, although simplified models are very useful to understand phenomena and dissect complex behaviors, they may induce misleading generalizations.

Despite passengers' acceleration attenuation, such low values may not be optimal choices for actual real-life models, since it can impair maneuvering. These low parameters also create the need to increase the suspension working space that may cause road-holding problems. Table 6 shows the working space for the selected solutions in comparison with that for the nominal values along with the DLC maneuvering. The increase of the working space is justified by the general decrease of the stiffness and damping parameters and happens to be a well-known behavior.

To confirm road-holding, the minimum peak values for tires at the Pareto front Point $G$ were evaluated and resulted in 21,004 N, 22,985 N, 38,859 N, 37,960 N for front right, front left, rear right, and rear left tires, respectively. The corresponding mean values are 32,435 N, $32,530 \mathrm{~N}, 57,054 \mathrm{~N}$, and 57,228 N. These mean and peak values are far away from negative values and indicate a constraint that is not active. For the other Pareto front solutions, the same was noticed, with none of the peak forces along time presenting negative values.

Figure 11 shows a typical acceleration time signal for the driver, for the nominal solution and solution $A$, respectively. The Crest factor showed to be lower than nine in all conditions, meaning that impact vibration is not an issue in this particular case. As one can see, the 
Table 7 Weighted RMS acceleration (ISO 2631-1 1997) for cases $A-H$ in (m/s ${ }^{2}$ )

\begin{tabular}{lllllllll}
\hline Pareto front case & $A$ & $B$ & $C$ & $D$ & $E$ & $F$ & $G$ & Nominal $H$ \\
\hline Driver (8 h) & 0.5659 & 0.5658 & 0.5658 & 0.5657 & 0.6438 & 0.7391 & 0.8446 & 0.8632 \\
Passenger 1 & 0.0321 & 0.0382 & 0.0458 & 0.0481 & 0.0325 & 0.0316 & 0.0310 & 0.0739 \\
Passenger 2 & 0.0485 & 0.0543 & 0.0789 & 0.0821 & 0.0487 & 0.0489 & 0.0497 & 0.0892 \\
\hline
\end{tabular}

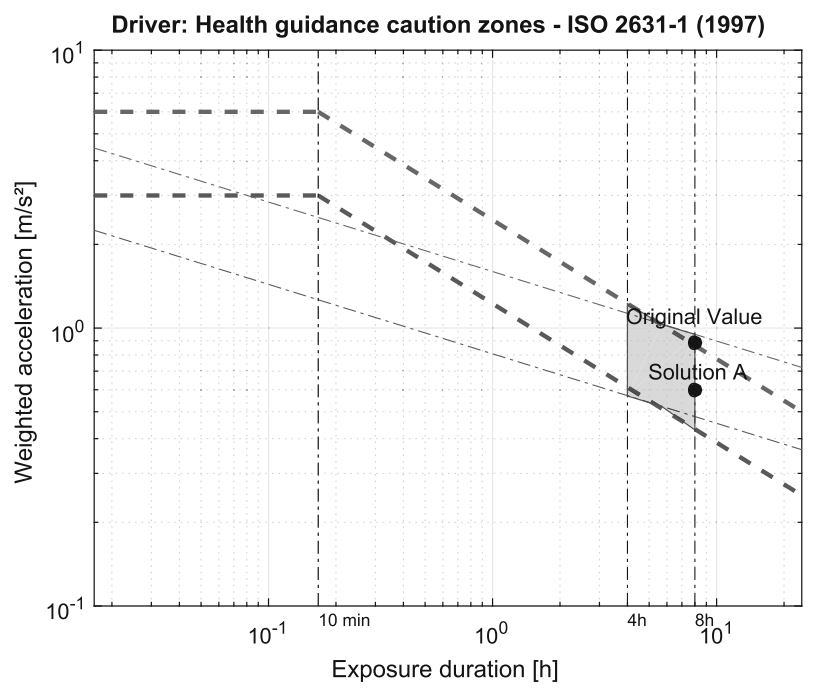

Fig. 12 Driver's Health guidance caution zones, ISO 2631-1 (1997). Comparison between solution $A$ and nominal parameter values

lateral acceleration originated by the DLC maneuver appears in the first $10 \mathrm{~s}$, although the road irregularities may disturb the overall trend. Moreover, it is also possible to notice that the vertical acceleration is greatly attenuated in the optimized solution.

Table 7 shows a comparison between the frequency-weighted RMS acceleration ( $\left.a_{\mathrm{wz} \text { rms }}\right)$ described in ISO 2631-1 (1997) from solution $A$ to $H$.

Figure 12 shows the improvement in the driver's health risk according to Standard ISO 2631-1 (1997) using optimized solution A.

According to Fig. 12, the driver acceleration is at the danger zone (Equation B.1, ISO 2631-1 1997) for a bus traveling under the specified conditions using nominal values for the suspension. For optimization A, the driver condition was shifted to the caution zone, at which there is no imminent health risk. The passengers were also benefited by the solution. The RMS acceleration reduction is significant and can be seen in Table 7. For comfort evaluation, the equivalent total acceleration takes into account different multiplying factors from those used in the health evaluation. Table 8 shows the corresponding equivalent total acceleration for driver and passengers.

From Tables 6 and 7, one can notice that although driver, passengers 1 and 2 were considered in a "not uncomfortable" situation for nominal parameters, their acceleration values were reduced by one-third and halved, respectively, at the optimized solution. 
Table 8 Equivalent total acceleration (ISO 2631-1 1997) for cases $A-H$ in $\left(\mathrm{m} / \mathrm{s}^{2}\right.$ )

\begin{tabular}{lllllllll}
\hline Pareto front case & $A$ & $B$ & $C$ & $D$ & $E$ & $F$ & $G$ & Nominal $H$ \\
\hline Driver & 0.0200 & 0.0200 & 0.0203 & 0.0203 & 0.0225 & 0.0256 & 0.0291 & 0.0298 \\
Passenger 1 & 0.0327 & 0.0387 & 0.0463 & 0.0486 & 0.0325 & 0.0323 & 0.0316 & 0.0742 \\
Passenger 2 & 0.0489 & 0.0547 & 0.0792 & 0.0824 & 0.0491 & 0.0493 & 0.0502 & 0.0895
\end{tabular}

Table 9 Vertical SEAT values (\%) for solution $A-H$ (reference)

\begin{tabular}{lrrrllllr}
\hline Pareto front case & \multicolumn{1}{c}{$A$} & \multicolumn{1}{c}{$B$} & \multicolumn{1}{c}{$C$} & \multicolumn{1}{c}{$D$} & $E$ & $F$ & $G$ & Nominal $H$ \\
\hline Driver & 38.8 & 38.9 & 38.4 & 38.3 & 43.6 & 51 & 55.1 & 50.4 \\
Passenger 1 & 109.0 & 123.5 & 151.2 & 157 & 109.3 & 109.6 & 111 & 210.5 \\
Passenger 2 & 126.6 & 149.4 & 157.1 & 164.1 & 126 & 125.1 & 122.8 & 282.6 \\
\hline
\end{tabular}

Table 9 shows a comparison between the SEAT values (Seat Effective Amplitude Transmissibility). Since the SEAT value is given by RMS vibration on the seat divided by vibration on the floor, one can conclude that the optimized case is best suited for vibration mitigation.

\section{Final remarks}

The market shows that passive suspension systems are still the most cost-effective approach nowadays for vibration mitigation, and thus, it is worth to be investigated. The parameter optimization of the vehicle's suspension can be a useful tool at the design phase mainly when multiple objectives are being considered at the same time. Misbehaved objective functions and the burden of computational costs pose a challenging task for optimization algorithms. The novel algorithm MOQPSO, proposed in this paper, has shown to be efficient at solving suspension optimization problems. In the half-car case, all the Pareto fronts resulted very similar to those presented in the literature. Specifically, in the case of a bi-objective problem $\boldsymbol{f}_{1} \times \boldsymbol{f}_{4}$, MOQPSO presented superior behavior when compared to the COGA-II algorithm, since non-dominated new regions were found. In the multi-objective optimization including all five objective functions, the results from the literature were covered by MOQPSO, since all projections show that those points are contained in the particle cloud or at least in the Pareto front. It is worth mentioning that COGA-II is an algorithm specially designed to solve high-dimensional multi-objective problems. Therefore, having a similar result using the MOQPSO in a five-dimensional multi-objective problem is an important validation for the proposed novel algorithm.

An enhanced suspension bus model that includes the lateral dynamics of the bus with a tire model and steering angles was proposed. A road profile with irregularities that follows ISO standard with correlations between tracks was also included in the simulations. The model was subjected to external excitations provided by the road irregularities and the maneuvering in a modified DLC test.

For the bus suspension optimization problem, all the solutions from the Pareto front found by NSGA-II were dominated by those found by MOQPSO, which also had a wider front for the same number of function calls. Once the optimization function calls were set constant for both algorithms, it is evident that MOQPSO presented a better efficiency than NSGA-II 
(advanced Pareto fronts and spread solutions). For future works, the performance of the new algorithm should be further tested, against other algorithms, using multi-objective benchmark functions and metrics.

The use of the algorithm in the bus test case showed improvements in vibration attenuation. For solution A, the driver was shifted from the vibration exposure limit zone to the vibration exposure action zone defined by ISO 2631-1 (1997). Similar improvements were obtained for passengers and driver's comfort, about $50 \%$ and $33 \%$, respectively. The main expected trends in optimized design variables using simple suspension models from literature, like small stiffness and damping to mitigate vibration, were observed in the examples. However, some obtained results did not endorse these trends. This shows the importance of elaborated vehicle suspension models that may unveil new behavior caused by the mutual interaction of different parts of the system.

For future works, new design variables can be elected, such as suspension geometric parameters, and tire pressure, as well as new objective functions, as working space and ground reaction forces. The effect of passenger's acceleration attenuation by increasing stiffness and damping of components at opposing regions of the suspension (Table 5, Fig. 10) is also a valid field for investigation. In future works, it seems plausible to compare performance between the optimized passive suspension and that with an active control system. The same framework presented in this paper can be used to optimize the parameters of the active $\mathrm{c}$ ontrol system such as location and limit values for the control forces or the gain parameters and will be subject to future investigations.

Funding Funding was provided by National Council for Scientific and Technological Development CNPq (Grant no. 301719-2017-9) and Coordenação de Aperfeiçoamento de Pessoal de Nível Superior (Grant no. 001).

\section{References}

Ahmadian MT, Sharifi SR and Abdollahpour R (2005) Application of car active suspension in a vertical acceleration reduction of vehicle due to road excitation and its effect on human health. In: 5 th International conference on multibody systems, nonlinear dynamics, and control, California, USA, vol 6, pp 375-382. ISBN: 0-7918-4806-X

Al-Baity H (2015) A quantum behaved particle swarm approach to multiobjective optimization. Ph.D. Thesis, The University of Birmingham

Boonlong K (2013) Multiobjective optimization of a vehicle vibration model using the improved compressedobjective genetic algorithm with convergence detection. Hindawi Publishing Corporation. Adv Mech Eng. https://doi.org/10.1155/2013/131495

Branke J, Mostaghim S (2006) About selecting the personal best in multiobjective particle swarm optimization. Parallel Probl Solv Nat PPSN IX 4196:523-532

Chen S, Shi T, Wang D, Chen J (2015) Multiobjective optimization of the vehicle ride comfort based on Kriging approximate model and NSGA-II. J Mech Sci Technol 29(3):1007-1018. https://doi.org/10.10 07/s12206-015-0215-x

Coello CAC, Lamont GB, van Veldhuizen DA (2007) Evolutionary algorithms for solving multiobjective problems-second edition. Springer, New York, pp 175-228 (ISBN: 978-0-387-33254-3)

Deb K, Pratap A, Agarwal S, Meyarivan T (2002) A fast and elitist multiobjective genetic algorithm: NSGA-II. IEEE Trans Evolut Comput. https://doi.org/10.1109/4235.996017

Fan Z, Wang TJ, Chen Z, Li G (2017) An improved multiobjective particle swarm optimization algorithm using minimum distance of point to line. Shock Vib 2017(5):1-16. https://doi.org/10.1155/2017/82048 67

Florea A, Cofaru II, Roamn L (2017) Applying the multiobjective optimization techniques in the design of suspension systems. J Dig Inf Manag 14(6):351-367

Gadhvia B, Savsania V, Patel V (2016) Multiobjective optimization of vehicle passive suspension system using NSGA-II, SPEA2 and PESA-II. Proc Technol 23:361-368. https://doi.org/10.1016/j.protcy.2016.03.038 
Goh CK, TanKC Liu DS, Chiam SC (2012) A competitive and cooperative co-evolutionary approach to multi-objective particle swarm optimization algorithm design. Eur J Oper Res 202:42-54

Gomes HM (2016) Multi-objective optimization of quarter car passive suspension design in the frequency domain based on PSO. Eng Comput Int J Comput Aided Eng Softw 33(5):1422-1423. https://doi.org/1 0.1108/EC-01-2015-0021

Griffin MJ (1990) Handbook of human vibration. Academic Press Limited, London. ISBN 978-0-12-303040-5

Hassani K, Lee W (2015) Multiobjective design of state feedback controllers using reinforced quantumbehaved particle swarm optimization. Appl Soft Comput 41:66-76. https://doi.org/10.1016/j.asoc.2015. 12.024

Havelka F, Musil M (2014) Multiobjective optimization of vehicle suspension parameters considering various road classes. In: Scientific proceedings 2014, Faculty of Mechanical Engineering, SUT in Bratislava, vol 22, pp 26-31. https://doi.org/10.2478/stu-2014-0005

ISO 8608:1995 (1995) Mechanical vibration-road surface profiles-reporting of measured data. ISO/TC108/SC2, International Organization for Standardization, Geneva, Switzerland

ISO 3888:1999 (1999) Test Track for a severe lane-change maneuver part 1: double lane change. ISO/TC 22/SC 33, International Organization for Standardization, Geneva, Switzerland

ISO 5982 (2001) Mechanical vibration and shock-Range of idealized values to characterize seated-body biodynamic response under vertical vibration. ISO/TC108/SC4, International Organization for Standardization, Geneva, Switzerland

ISO 2631-1:1997 (1997) Mechanical vibration and shock-evaluation of human exposure to whole-body vibration. ISO/TC108/SC4, International Organization for Standardization, Geneva, Switzerland

Ji X, Eger TR, Dickey JP (2017) Evaluation of the vibration attenuation properties of an air-inflated cushion with two different heavy machinery seats in multi-axis vibration environments including jolts. Appl Ergon 59:293-301. https://doi.org/10.1016/j.apergo.2016.06.011

Knowles J, Corne D (1999) The Pareto archived evolution strategy: a new baseline algorithm for Pareto multiobjective optimisation. In: Proceedings of the 1999 congress on evolutionary computation, 1999. (CEC 99), vol 1. IEEE 1999. https://doi.org/10.1109/cec.1999.781913

Koulocheris D, Papaioannou G, Christodoulou D (2017) An approach for multiobjective optimization of vehicle suspension system. IOP Conf Ser Mater Sci Eng. https://doi.org/10.1088/1757-899x/252/1/012 037

Lee CK, Cheng YC (2014) Application of uniform design and QPSO in the sensitivity problem a railway vehicle system. Proc Eng 79:427-436. https://doi.org/10.1016/j.proeng.2014.06.364

Mahmoodabadi MJ, Safaie A, Bagheri A, Nariman-zadeh N (2013) A novel combination of Particle Swarm Optimization and Genetic Algorithm for Pareto optimal design of a five-degree of freedom vehicle vibration model. Appl Soft Comput 13:2577-2591. https://doi.org/10.1016/j.asoc.2012.11.028

Mitra CA, Desai JG, Patwardhan RS, Shirke HP, Kurne MHW, Banerjee N (2016) Optimization of passive vehicle suspension system by genetic algorithm. Proc Eng 144:1158-1166

Nariman-Zadeh N, Salehpour M, Jamali A, Haghgoo E (2010) Pareto optimization of a five-degree of freedom vehicle vibration model using a multi-objective uniform-diversity genetic algorithm (MUGA). Eng Appl Artif Intell 23:543-551. https://doi.org/10.1016/j.engappai.2009.08.008

Nawayseh N (2015) Effect of the seating condition on the transmission of vibration through the seat pan and backrest. Int J Ind Ergon 45:82-90. https://doi.org/10.1016/j.ergon.2014.12.005

Omkar SN, Khanderlwal R, Ananth TVS, Narayana NG, Gopalakrishnan S (2009) Quantum behaved Particle Swarm Optimization (QPSO) for multiobjective design optimization of composite structures. Expert Syst Appl 36:11312-11322. https://doi.org/10.1016/j.eswa.2009.03.006

Rill G (2012) Road vehicle dynamics: fundamentals and modeling, 1a edn. Taylor and Francis Group, Boca Raton

Sammonds GM, Fray M, Mansfield NJ (2017) Effect of long term driving on driver discomfort and its relationship with seat fidgets and movements. Appl Ergon 58:119-127. https://doi.org/10.1016/j.apergo. 2016.05.009

Sekulic D, Dedovic V, Rusov S, Salinic S, Obradovic A (2013) Analysis of vibration effects on the comfort of intercity bus users by oscillatory model with ten degrees of freedom. Appl Math Model 37:8629-8644

Sert E, Boyraz P (2017) Optimization of suspension system and sensitivity analysis for improvement of stability in a midsize heavy vehicle. Eng Sci Technol Int J 20(2017):997-1012

Sharp RS, Crolla DA (1987) Road vehicle suspension system design—a review. Veh Syst Dyn Int J Veh Mech Mobil 16(3):167-192. https://doi.org/10.1080/00423118708968877

Spentzas K, Kanarachos SA (2002) Design of a non-linear hybrid car suspension system using neural networks. Math Comput Simul 60(3-5):369-378. https://doi.org/10.1016/S0378-4754(02)00029-0

Sun J, Feng B and Xu W (2004) Particle swarm optimization with particles having quantum behavior. In: Proceedings of congress on evolutionary computation, Portland, OR, USA, pp 325-331 
Sun J, Xu W, Feng B (2004) A global search strategy of quantum-behaved particle swarm optimization. In: Cybernetics and intelligent systems, 2004 IEEE conference, vol 1, pp 111-116

Sun J, Lai C-H, Wu X-J (2011) Particle Swarm Optimization: classical and quantum perspectives. CRC Press Ed, Chapman \& Hall/CRC Numerical Analysis and Scientific Computing Series

Xu S-H, Mu X-D, Chai D, Zhao P (2016) Multiobjective quantum-behaved particle swarm optimization algorithm with double potential well and share learning. Optik 127:4921-4927. https://doi.org/10.1016/ j.ijleo.2016.02.049

Yang X, Shi P, Shen W, Jiang K, Pang S (2013) Multiobjective quantum-behaved particle swarm optimization with entropy-based density assessment and chaotic mutation operator. J Comput Inf Syst 9(10):3873-3881

Zamanian A, Nikravesh A, Monazzam MR, Hassanzadeh J, Fararouei M (2014) Short-term exposure with vibration and its effect on attention. J Environ Health Sci Eng 12(1):135. https://doi.org/10.1186/s4020 1-014-0135-1

Zhan S, Qing-Wei C (2011) Multi-objective quantum-behaved particle swarm optimization algorithm based on QPSO and crowding distance sorting. Control Decis 26(4):540-547

Zhang X, Qiu Y, Griffin MJ (2015) Transmission of vertical vibration through a seat: effect of thickness of foam cushions at the seat pan and the backrest. Int J Ind Ergon 48:36-45. https://doi.org/10.1016/j.ergon. 2015.03.006

Zhou D-Z, Li Y, Jiang B, Wang J (2016) A novel multiobjective quantum-behaved particle swarm optimization based on the ring model. Math Probl Eng 2016:1-15. https://doi.org/10.1155/2016/4968938

Publisher's Note Springer Nature remains neutral with regard to jurisdictional claims in published maps and institutional affiliations. 